QUARTERLY OF APPLIED MATHEMATICS

VOLUME LXVII, NUMBER 1

MARCH 2009, PAGES 163-193

S 0033-569X(09)01125-0

Article electronically published on January 22, 2009

\title{
ASYMMETRIC AND MOVING-FRAME APPROACHES TO NAVIER-STOKES EQUATIONS
}

\author{
BY \\ XIAOPING XU \\ Institute of Mathematics, Academy of Mathematics 8 System Sciences Chinese Academy of Sciences,
} Beijing 100190, People's Republic of China

Abstract. In this paper, we introduce a method of imposing asymmetric conditions on the velocity vector with respect to independent variables and a method of moving frame for solving the three-dimensional Navier-Stokes equations. Seven families of nonsteady rotating asymmetric solutions with various parameters are obtained. In particular, one family of solutions blows up at any point on a moving plane with a line deleted, which may be used to study turbulence. Using Fourier expansion and two families of our solutions, one can obtain discontinuous solutions that may be useful in the study of shock waves. Another family of solutions are partially cylindrical invariant, contain two parameter functions of $t$ and structurally depend on two arbitrary polynomials, which may be used to describe incompressible fluid in a nozzle. Most of our solutions are globally analytic with respect to spacial variables.

1. Introduction. The most fundamental differential equations in the motion of incompressible viscous fluid are Navier-Stokes equations:

$$
\begin{aligned}
u_{t}+u u_{x}+v u_{y}+w u_{z}+\frac{1}{\rho} p_{x} & =\nu\left(u_{x x}+u_{y y}+u_{z z}\right), \\
v_{t}+u v_{x}+v v_{y}+w v_{z}+\frac{1}{\rho} p_{y} & =\nu\left(v_{x x}+v_{y y}+v_{z z}\right), \\
w_{t}+u w_{x}+v w_{y}+w w_{z}+\frac{1}{\rho} p_{z} & =\nu\left(w_{x x}+w_{y y}+w_{z z}\right), \\
u_{x}+v_{y}+w_{z} & =0,
\end{aligned}
$$

where $(u, v, w)$ stands for the velocity vector of the fluid, $p$ stands for the pressure of the fluid, $\rho$ is the density constant and $\nu$ is the coefficient constant of the kinematic viscosity.

Received September 5, 2007.

2000 Mathematics Subject Classification. Primary 35C05, 35Q35; Secondary 35C10, 35C15.

Key words and phrases. Navier-Stokes equations, asymmetric condition, moving frame, exact solution, symmetry transformation.

Research for this article was supported by China NSF Grant \#10871193.

E-mail address: xiaoping@math.ac.cn

(C)2009 Brown University 
The Lie point symmetries of the two-dimensional special case of the above equations $\left(u_{z}=v_{z}=w=0\right)$ were obtained by Pukhnachev Pv1 and Buchnev Ba]. Moreover, certain group-invariant solutions were found in the works of Pukhnachev Pv1, KochinKibel'-Roze [KKR] and Bytev Bv1, Bv2. Futhermore, Gryn G] obtained a certain exact solution describing flows between porous walls in the presence of injection and suction at identical rates, and Polyanin $\mathrm{Pa}$ used the method of generalized separation of variables to find certain exact solutions.

Assuming nullity of certain components of the tensor of momentum flow density, Landau [Ll] found a exact solution of Navier-Stokes equations (1.1)-(1.4), which describes an axially symmetrical jet discharging from a thin pipe into unbounded space. The Lie point symmetries of the above three-dimensional equations were obtained by Buchnev [Ba and Pukhnachev [Pv2]. Moreover, Kapitanskii [K] found certain cylindrical invariant solutions of the equations, and Yakimov $[\mathrm{Y}]$ obtained exact solutions with a singularity of the type of a vortex filament situated on a half line. Shen [S1, S2] rewrote Navier-Stokes equations in terms of complex variables and found certain exact solutions. Brutyan and Karapivskii $[\mathrm{BK}]$ got exact solutions describing the evolution of a vortex structure in a generalized shear flow. Furthermore, Leipnik $[\mathrm{Lr}$ obtained exact solutions by a recursive series of diffusive quotients, and Vyskrebtsov [V] studied self-similar solutions for an axisymmetric flow of a viscous incompressible flow.

From an algebraic point of view, it seems to us that there are not enough exact solutions that fully reflect the fundamental natures of Navier-Stokes equations. In this paper, we introduce a method of imposing asymmetric conditions on the velocity vector with respect to independent variables and obtain two families of non-steady asymmetric solutions with rotation. One of the families contains two arbitrary parameter functions of $t$ and an arbitrary number of parameter constants. Using Fourier expansion and this family of solutions, one can obtain discontinuous solutions that may be useful in the study of shock waves. Another family of solutions is partially cylindrical invariant, contains two parameter functions of $t$ and structurally depends on two arbitrary polynomials, which may be used to describe incompressible fluid in a nozzle. In order to better reflect the rotating nature of flow, we also give a method of moving frame and find five families of non-steady rotating solutions with various parameters. In particular, one family of solutions blows up at any point on a moving plane with a line deleted, which may be used to study turbulence. Another family can be used to obtain discontinuous rotating solutions. Most of our solutions are globally analytic with respect to spacial variables. Below we give a more detailed introduction.

The equations (1.1)-(1.4) are invariant under orthogonal transformations $\left\{T_{A} \mid A \in\right.$ $O(n, \mathbb{R})\}$ with

$$
T_{A}\left[\left(\begin{array}{l}
x \\
y \\
z
\end{array}\right)\right]=A\left(\begin{array}{l}
x \\
y \\
z
\end{array}\right), T_{A}\left[\left(\begin{array}{c}
u \\
v \\
w
\end{array}\right)\right]=A\left(\begin{array}{c}
u \\
v \\
w
\end{array}\right), T_{A}(p)=p .
$$


Moreover, they are invariant under the time translation $t \mapsto t+a$ with $a \in \mathbb{R}$ and the scaling $T_{b}$ with $0 \neq b \in \mathbb{R}$ :

$$
\begin{array}{ll}
T_{b}(u)=b^{-1} u\left(b^{2} t, b x, b y, b z\right), & T_{b}(v)=b^{-1} v\left(b^{2} t, b x, b y, b z\right), \\
T_{b}(w)=b^{-1} w\left(b^{2} t, b x, b y, b z\right), & T_{b}(p)=b^{-2} p\left(b^{2} t, b x, b y, b z\right) .
\end{array}
$$

The most interesting symmetries of Navier-Stokes equations are the following timedependent translations:

$$
\begin{aligned}
T_{1 \alpha}(u) & =u(t, x+\alpha, y, z)-\alpha^{\prime}, \quad T_{1 \alpha}(v)=v(t, x+\alpha, y, z), \\
T_{1 \alpha}(w) & =w(t, x+\alpha, y, z), \quad T_{1 \alpha}(p)=p(t, x+\alpha, z)+\rho \alpha^{\prime \prime} x
\end{aligned}
$$

and its permutations on $(u, x),(v, y),(w, z)$, and

$$
T_{2 \alpha}(u)=u, \quad T_{2 \alpha}(v)=v, \quad T_{2 \alpha}(w)=w, \quad T_{2 \alpha}(p)=p+\alpha,
$$

where $\alpha$ is an arbitrary function of $t$. The above transformations transform solutions of Navier-Stokes equations into their solutions. Our goal in this paper is to find exact solutions of Navier-Stokes equations modulo the above symmetries. In other words, the above symmetries will be used to simplify our ansatzes for exact solutions and related arguments.

For convenience, we always assume that all the involved partial derivatives of related functions always exist and we can change the order of taking partial derivatives. In fluid dynamics, rotation-free solutions of Navier-Stokes equations, namely,

$$
u_{y}-v_{x}=0, \quad v_{z}-w_{y}=0, \quad w_{x}-u_{z}=0,
$$

are not so interesting. From a pure mathematical point of view, a rotation-free solution is equivalent to a time-dependent harmonic function $f(t, x, y, x)$ (i.e., $f_{x x}+f_{y y}+f_{z z}=0$ ), where

$$
u=f_{x}, \quad v=f_{y}, \quad w=f_{z} .
$$

Practically, steady solutions (or time-independent) are not very important. In general, it is difficult to find exact non-steady rotating solutions for Navier-Stokes equations (1.1)(1.4) due to their non-linearity.

Using a certain finite-dimensional stable range of the non-linear term, we found in [X1] a family of exact solutions with seven parameter functions for the equation of nonstationary transonic gas flows found by Lin, Reisner and Tsien LRT, which blow up on a moving line. These solutions may reflect partial phenomena of gust. In [X2], we use various ansatzes with undermined functions and the technique of moving frame to find basic solutions modulo the Lie point symmetries with parameter functions for the classical non-steady boundary layer problems. These two works motivated us to solve Navier-Stokes equations by algebraic methods.

Our first idea is to impose suitable asymmetric conditions on the velocity vector with respect to independent variables. For instance, assuming

$$
\begin{gathered}
u=\gamma(t) x+y \phi\left(t, x^{2}+y^{2}\right), \quad v=\gamma(t) y-x \phi\left(t, x^{2}+y^{2}\right), \\
w=\psi\left(t, x^{2}+y^{2}\right)-2 \gamma(t) z,
\end{gathered}
$$


we obtain the following solution of Navier-Stokes equations (see Theorem 2.4):

$$
\begin{gathered}
u=\frac{\alpha^{\prime}}{2 \alpha} x+\frac{\beta y}{x^{2}+y^{2}}+y \sum_{i=0}^{\infty} \frac{\left(\alpha \partial_{t}\right)^{i}(\Im)}{i !(i+1) ! \alpha}\left(\frac{x^{2}+y^{2}}{4 \nu \alpha}\right)^{i}, \\
v=\frac{\alpha^{\prime}}{2 \alpha} y-\frac{\beta x}{x^{2}+y^{2}}-x \sum_{i=0}^{\infty} \frac{\left(\alpha \partial_{t}\right)^{i}(\Im)}{i !(i+1) ! \alpha}\left(\frac{x^{2}+y^{2}}{4 \nu \alpha}\right)^{i}, \\
w=\alpha \sum_{s=0}^{\infty} \frac{\left(\alpha \partial_{t}\right)^{s}(\varphi)}{(s !)^{2}}\left(\frac{x^{2}+y^{2}}{4 \nu \alpha}\right)^{s}-\frac{\alpha^{\prime}}{\alpha} z, \\
p=\frac{\rho\left(\left(\alpha^{\prime}\right)^{2}-2 \alpha \alpha^{\prime \prime}\right)\left(x^{2}+y^{2}\right)}{8 \alpha^{2}}+\frac{\rho\left(\alpha \alpha^{\prime \prime}-2\left(\alpha^{\prime}\right)^{2}\right) z^{2}}{\alpha^{2}}+\beta^{\prime} \arctan \frac{y}{x} \\
+2 \nu \rho \alpha \sum_{i, s=0}^{\infty} \frac{\left[\left(\alpha \partial_{t}\right)^{i}(\Im)\right]\left[\left(\alpha \partial_{t}\right)^{s}(\varphi)\right]}{i !(i+1) ! s !(s+1) !(\alpha)^{2}}\left(\frac{x^{2}+y^{2}}{4 \nu \alpha}\right)^{i+s+1},
\end{gathered}
$$

where $\alpha, \beta$ are any functions in $t$ and $\Im, \varphi$ are arbitrary polynomials in $t$. The above solution can be used to describe incompressible fluid in a nozzle. The polynomials $\Im$ and $\varphi$ can be replaced by the other functions as long as the related power series converge.

As we emphasized earlier, people are interested in solutions that are not rotation free. To better capture the rotating nature of fluid, we introduce the following moving frames:

$$
\begin{gathered}
\mathcal{X}=x \cos \alpha+(y \cos \beta+z \sin \beta) \sin \alpha, \quad \mathcal{Y}=-x \sin \alpha+(y \cos \beta+z \sin \beta) \cos \alpha, \\
\mathcal{Z}=-y \sin \beta+z \cos \beta, \quad \mathcal{U}=u \cos \alpha+(v \cos \beta+w \sin \beta) \sin \alpha, \\
\mathcal{V}=-u \sin \alpha+(v \cos \beta+w \sin \beta) \cos \alpha, \quad \mathcal{W}=-v \sin \beta+w \cos \beta
\end{gathered}
$$

where $\alpha$ and $\beta$ are arbitrary functions in $t$. Here we exclude the translation components because Navier-Stokes equations are invariant under the transformations of the type $T_{1 \alpha}$ in (1.8) and (1.9), and we want to consider solutions modulo these transformations. With respect to the above rotating frames, Navier-Stokes equations change to a more complicated system of partial differential equations. Imposing asymmetric conditions on the moving frames, we find another five families of non-steady rotating solutions with various parameters. For instance, we have the following solution of Navier-Stokes 
equations (see Theorem 3.3):

$$
\begin{aligned}
& u=\left(\frac{\alpha^{\prime \prime}}{2 \alpha^{\prime}}+6 \nu \mathcal{Y} \mathcal{X}^{-2}\right)(\mathcal{Y} \sin \alpha-\mathcal{X} \cos \alpha)-\alpha^{\prime}(\mathcal{X} \sin \alpha+\mathcal{Y} \cos \alpha), \\
& v=-\left(\frac{\alpha^{\prime \prime}}{2 \alpha^{\prime}}+6 \nu \mathcal{Y} \mathcal{X}^{-2}\right)(\mathcal{X} \sin \alpha+\mathcal{Y} \cos \alpha) \cos \beta \\
&+\alpha^{\prime}(\mathcal{X} \cos \alpha-\mathcal{Y} \sin \alpha) \cos \beta-\beta^{\prime} \mathcal{Z} \cos \beta \\
&+\left(\beta^{\prime} \mathcal{X} \sin \alpha+\beta^{\prime} \mathcal{Y} \cos \alpha-\frac{\alpha^{\prime \prime}}{\alpha^{\prime}} \mathcal{Z}\right) \sin \beta, \\
& w=-\left(\frac{\alpha^{\prime \prime}}{2 \alpha^{\prime}}+6 \nu \mathcal{Y} \mathcal{X}^{-2}\right)(\mathcal{X} \sin \alpha+\mathcal{Y} \cos \alpha) \sin \beta \\
&+\alpha^{\prime}(\mathcal{X} \cos \alpha-\mathcal{Y} \sin \alpha) \sin \beta-\beta^{\prime} \mathcal{Z} \sin \beta \\
&+\left(\frac{\alpha^{\prime \prime}}{\alpha^{\prime}} \mathcal{Z}-\beta^{\prime} \mathcal{X} \sin \alpha-\beta^{\prime} \mathcal{Y} \cos \alpha\right) \cos \beta, \\
& p=\left\{\frac{\left(2 \alpha^{\prime} \alpha^{\prime \prime \prime}+\right.}{+}\left(\alpha^{\prime}\right)^{4}-3\left(\alpha^{\prime \prime}\right)^{2}\right)\left(\mathcal{X}^{2}+\mathcal{Y}^{2}\right) 8\left(\alpha^{\prime}\right)^{2}-\frac{3\left(\beta^{\prime}\right)^{2}\left(\mathcal{X}^{2} \sin ^{2} \alpha+\mathcal{Y}^{2} \cos ^{2} \alpha\right)}{2} \\
&+12 \nu\left(\alpha^{\prime} \mathcal{Y} \mathcal{X}^{-1}-\nu \mathcal{X}^{-2}\right)+\left(\beta^{\prime \prime}-4 \beta^{\prime} \gamma\right) \mathcal{Z}(\mathcal{X} \sin \alpha+\mathcal{Y} \cos \alpha) \\
&\left.-3\left(\beta^{\prime}\right)^{2} \mathcal{X} \mathcal{Y} \sin \alpha \cos \alpha+\frac{\left(\alpha^{\prime} \alpha^{\prime \prime \prime}+\left(\alpha^{\prime}\right)^{2}\left(\beta^{\prime}\right)^{2}-2\left(\alpha^{\prime \prime}\right)^{2}\right) \mathcal{Z}^{2}}{2\left(\alpha^{\prime}\right)^{2}}\right\}
\end{aligned}
$$

The above solution blows up at any point on the following rotating plane with a line deleted:

$$
\begin{aligned}
& \left\{(x, y, z) \in \mathbb{R}^{3} \mid x \cos \alpha+y \sin \alpha \cos \beta+z \sin \alpha \sin \beta=0,\right. \\
& -x \sin \alpha+y \cos \alpha \cos \beta+z \cos \alpha \sin \beta \neq 0\} .
\end{aligned}
$$

This type of solution may be applied in studying turbulence. Since all of our solutions in this paper only involve elementary functions and integrations, they may be applied to engineering problems with the help of a computer, although they appear sophisticated in format. They can also be used to solve certain initial value problems for Navier-Stokes equations because they contain parameter functions.

As we all know that, in general, it is impossible to find all the solutions of nonlinear partial differential equations both analytically and algebraically. In our arguments throughout this paper, we always search for reasonable sufficient conditions of obtaining exact solutions. For instance, we treat non-zero functions like "non-zero constants" for this purpose because our approaches in this paper are completely algebraic. Of course, one can use our methods in this paper to get more solutions, in particular, by considering the support and discontinuity of the related functions. We want to remind the reader that we always put arguments (proofs) before our theorems (conclusions) due to our purpose of finding exact solutions.

The paper is organized as follows. Section 2 is devoted to our asymmetric approaches. We present the general settings for the moving-frame approach in Section 3 and find two families of exact solutions. In Section 4, we use the moving frames and certain ansatzes involving given irrational functions to find another three families of exact solutions. 
2. Asymmetric approaches. In this section, we will solve incompressible NavierStokes equations (1.1)-(1.4) by imposing asymmetric assumptions on $u, v$ and $w$.

For convenience of computation, we denote

$$
\begin{gathered}
\Phi_{1}=u_{t}+u u_{x}+v u_{y}+w u_{z}-\nu\left(u_{x x}+u_{y y}+u_{z z}\right), \\
\Phi_{2}=v_{t}+u v_{x}+v v_{y}+w v_{z}-\nu\left(v_{x x}+v_{y y}+v_{z z}\right), \\
\Phi_{3}=w_{t}+u w_{x}+v w_{y}+w w_{z}-\nu\left(w_{x x}+w_{y y}+w_{z z}\right) .
\end{gathered}
$$

Then Navier-Stokes equations become

$$
\Phi_{1}+\frac{1}{\rho} p_{x}=0, \quad \Phi_{2}+\frac{1}{\rho} p_{y}=0, \quad \Phi_{3}+\frac{1}{\rho} p_{z}=0
$$

and $u_{x}+v_{y}+w_{z}=0$. Our strategy is the first to solve the following compatibility conditions:

$$
\partial_{y}\left(\Phi_{1}\right)=\partial_{x}\left(\Phi_{2}\right), \quad \partial_{z}\left(\Phi_{1}\right)=\partial_{x}\left(\Phi_{3}\right), \quad \partial_{z}\left(\Phi_{2}\right)=\partial_{y}\left(\Phi_{3}\right)
$$

and then find $p$ via (2.4).

Let us first look for the simplest non-steady solutions of Navier-Stokes equations (indeed, the corresponding Euler equations) that are not rotation free. This will help the reader to better understand our later approaches. Assume

$$
u=\gamma_{1} x-\alpha_{1} y-\alpha_{2} z, \quad v=\alpha_{1} x+\gamma_{2} y-\alpha_{3} z, \quad w=\alpha_{2} x+\alpha_{3} y+\gamma_{3} z,
$$

where $\alpha_{i}$ and $\gamma_{i}$ are functions in $t$ such that $\gamma_{1}+\gamma_{2}+\gamma_{3}=0$. Then

$$
\begin{aligned}
& \Phi_{1}=\left(\gamma_{1}^{\prime}+\gamma_{1}^{2}-\alpha_{1}^{2}-\alpha_{2}^{2}\right) x-\left(\alpha_{1}^{\prime}-\alpha_{1} \gamma_{3}+\alpha_{2} \alpha_{3}\right) y+\left(\alpha_{1} \alpha_{3}-\alpha_{2}^{\prime}+\alpha_{2} \gamma_{2}\right) z, \\
& \Phi_{2}=\left(\alpha_{1}^{\prime}-\alpha_{1} \gamma_{3}-\alpha_{2} \alpha_{3}\right) x+\left(\gamma_{2}^{\prime}+\gamma_{2}^{2}-\alpha_{1}^{2}-\alpha_{3}^{2}\right) y-\left(\alpha_{3}^{\prime}+\alpha_{1} \alpha_{2}-\alpha_{3} \gamma_{1}\right) z, \\
& \Phi_{3}=\left(\alpha_{2}^{\prime}+\alpha_{1} \alpha_{3}-\alpha_{2} \gamma_{2}\right) x+\left(\alpha_{3}^{\prime}-\alpha_{1} \alpha_{2}-\alpha_{3} \gamma_{1}\right) y+\left(\gamma_{3}^{\prime}+\gamma_{3}^{2}-\alpha_{2}^{2}-\alpha_{3}^{2}\right) z .
\end{aligned}
$$

Furthermore,

$$
\begin{aligned}
& \partial_{y}\left(\Phi_{1}\right)=\partial_{x}\left(\Phi_{2}\right) \Longrightarrow \gamma_{3}=\frac{\alpha_{1}^{\prime}}{\alpha_{1}}, \\
& \partial_{z}\left(\Phi_{1}\right)=\partial_{x}\left(\Phi_{3}\right) \Longrightarrow \gamma_{2}=\frac{\alpha_{2}^{\prime}}{\alpha_{2}}, \\
& \partial_{z}\left(\Phi_{2}\right)=\partial_{y}\left(\Phi_{3}\right) \Longrightarrow \gamma_{1}=\frac{\alpha_{3}^{\prime}}{\alpha_{3}} .
\end{aligned}
$$

Note that

$$
\gamma_{1}+\gamma_{2}+\gamma_{3}=0 \sim \frac{\alpha_{1}^{\prime}}{\alpha_{1}}+\frac{\alpha_{2}^{\prime}}{\alpha_{2}}+\frac{\alpha_{3}^{\prime}}{\alpha_{3}}=0 \sim \alpha_{1} \alpha_{2} \alpha_{3}=c
$$

for some real constant $c$. Moreover,

$$
\begin{gathered}
\Phi_{1}=\left(\alpha_{3}^{\prime \prime} \alpha_{3}^{-1}-\alpha_{1}^{2}-\alpha_{2}^{2}\right) x-\alpha_{2} \alpha_{3} y+\alpha_{1} \alpha_{3} z, \\
\Phi_{2}=-\alpha_{2} \alpha_{3} x+\left(\alpha_{2}^{\prime \prime} \alpha_{2}^{-1}-\alpha_{1}^{2}-\alpha_{3}^{2}\right) y-\alpha_{1} \alpha_{2} z, \\
\Phi_{3}=\alpha_{1} \alpha_{3} x-\alpha_{1} \alpha_{2} y+\left(\alpha_{1}^{\prime \prime} \alpha_{1}^{-1}-\alpha_{2}^{2}-\alpha_{3}^{2}\right) .
\end{gathered}
$$


By (2.4),

$$
\begin{aligned}
p & =\frac{\rho}{2}\left[\left(\alpha_{1}^{2}+\alpha_{2}^{2}-\alpha_{3}^{\prime \prime} \alpha_{3}^{-1}\right) x^{2}+\left(\alpha_{1}^{2}+\alpha_{3}^{2}-\alpha_{2}^{\prime \prime} \alpha_{2}^{-1}\right) y^{2}\right. \\
& \left.+\left(\alpha_{2}^{2}+\alpha_{3}^{2}-\alpha_{1}^{\prime \prime} \alpha_{1}^{-1}\right) z^{2}\right]+\rho\left(\alpha_{2} \alpha_{3} x y-\alpha_{1} \alpha_{3} x z+\alpha_{1} \alpha_{2} y z\right)
\end{aligned}
$$

modulo the transformation in (1.10).

Proposition 2.1. Let $\alpha_{1}, \alpha_{2}$ and $\alpha_{3}$ be functions in $t$ such that $\alpha_{1} \alpha_{2} \alpha_{3}=c$ for some real constant $c$. Then we have the following solution of Navier-Stokes equations (1.1)-(1.4):

$$
u=\frac{\alpha_{3}{ }^{\prime}}{\alpha_{3}} x-\alpha_{1} y-\alpha_{2} z, \quad v=\alpha_{1} x+\frac{\alpha_{2}{ }^{\prime}}{\alpha_{2}} y-\alpha_{3} z, \quad w=\alpha_{2} x+\alpha_{3} y+\frac{\alpha_{1}{ }^{\prime}}{\alpha_{1}} z
$$

and $p$ is given in (2.17).

Next we assume

$$
v=-\frac{\beta^{\prime \prime}}{2 \beta^{\prime}} y, \quad w=\psi(t, z),
$$

where $\beta$ is a function in $t, \psi$ is a function of $t, z$ and $v$ is so written just for computational convenience by our earlier experience in [X1, X2]. According to (1.4),

$$
u=f(t, y, z)+\left(\frac{\beta^{\prime \prime}}{2 \beta^{\prime}}-\psi_{z}\right) x
$$

for some function $f$ of $t, y, z$. Then

$$
\begin{aligned}
\Phi_{1} & =f_{t}+f\left(\frac{\beta^{\prime \prime}}{2 \beta^{\prime}}-\psi_{z}\right)-\frac{\beta^{\prime \prime}}{2 \beta^{\prime}} y f_{y}+\psi f_{z}-\nu\left(f_{y y}+f_{z z}\right) \\
& +\left[\left(\frac{\beta^{\prime \prime}}{2 \beta^{\prime}}-\psi_{z}\right)^{2}+\frac{\beta^{\prime}{\beta^{\prime \prime}}^{\prime \prime}-\left(\beta^{\prime \prime}\right)^{2}}{2\left(\beta^{\prime}\right)^{2}}-\psi_{z t}-\psi \psi_{z z}+\nu \psi_{z z z}\right] x \\
\Phi_{2} & =\frac{\left(3\left(\beta^{\prime \prime}\right)^{2}-2 \beta^{\prime}{\beta^{\prime \prime}}^{\prime \prime}\right) y}{4\left(\beta^{\prime}\right)^{2}}, \quad \Phi_{3}=\psi_{t}+\psi \psi_{z}-\nu \psi_{z z} .
\end{aligned}
$$

Thus (2.5) is equivalent to the following equations:

$$
\begin{gathered}
\mathcal{T}\left[f_{t}+f\left(\frac{\beta^{\prime \prime}}{2 \beta^{\prime}}-\psi_{z}\right)-\frac{\beta^{\prime \prime}}{2 \beta^{\prime}} y f_{y}+\psi f_{z}-\nu\left(f_{y y}+f_{z z}\right)\right]=0 \\
\mathcal{T}\left[\psi_{z}^{2}-\frac{\beta^{\prime \prime}}{\beta^{\prime}} \psi_{z}-\psi_{z t}-\psi \psi_{z z}+\nu \psi_{z z z}\right]=0
\end{gathered}
$$

with $\mathcal{T}=\partial_{y}, \partial_{z}$.

Given $b, c \in \mathbb{R}$ and a function $\gamma$ of $t$, we set

$$
\begin{array}{ll}
\xi_{0}=b e^{\sqrt{\gamma^{\prime}} z+\nu \gamma}-c e^{-\sqrt{\gamma^{\prime}} z-\nu \gamma}, & \xi_{1}=b \sin \left(\sqrt{\gamma^{\prime}} z-\nu \gamma\right), \\
\zeta_{0}=b e^{\sqrt{\gamma^{\prime}} z+\nu \gamma}+c e^{-\sqrt{\gamma^{\prime}} z-\nu \gamma}, & \zeta_{1}=b \cos \left(\sqrt{\gamma^{\prime}} z-\nu \gamma\right) .
\end{array}
$$

Then we have the following solution of (2.24):

$$
\psi=\frac{\xi_{r}}{\beta^{\prime} \sqrt{\left(\gamma^{\prime}\right)^{3}}}-\frac{\gamma^{\prime \prime}}{2 \gamma^{\prime}} z
$$


with $r=0,1$. Write

$$
f=\frac{\hat{f}}{\sqrt{\beta^{\prime} \gamma^{\prime}}}
$$

Equation (2.23) is implied by the following equation:

$$
\hat{f}_{t}-\frac{\hat{f} \zeta_{r}}{\beta^{\prime} \gamma^{\prime}}-\frac{\beta^{\prime \prime}}{2 \beta^{\prime}} y \hat{f}_{y}-\frac{\gamma^{\prime \prime}}{2 \gamma^{\prime}} z \hat{f}_{z}+\frac{\hat{f}_{z} \xi_{r}}{\beta^{\prime} \sqrt{\left(\gamma^{\prime}\right)^{3}}}-\nu\left(\hat{f}_{y y}+\hat{f}_{z z}\right)=0
$$

To solve the above equation, we assume

$$
\hat{f}=h(t, y)+g(t, y) \zeta_{r},
$$

where $h$ and $g$ are functions in $t, y$. Then (2.29) is implied by the following two equations:

$$
\begin{gathered}
g_{t}-\frac{\beta^{\prime \prime}}{2 \beta^{\prime}} y g_{y}-\nu g_{y y}-\frac{h}{\beta^{\prime} \gamma^{\prime}}=0, \\
h_{t}-\frac{\beta^{\prime \prime}}{2 \beta^{\prime}} y h_{y}-\nu h_{y y}-\frac{\left(4 \delta_{0, r} b c+\delta_{1, r} b^{2}\right) g}{\beta^{\prime} \gamma^{\prime}}=0 .
\end{gathered}
$$

We will solve the above system of partial differential equations according to the following two cases:

Case 1. $r=0, b c=0$ or $r=1, b=0$.

In this case, we have the following solutions:

$$
\begin{gathered}
g=\sum_{i=1}^{m} d_{1, i} e^{\nu\left(a_{1, i}^{2}-b_{1, i}^{2}\right) \beta+a_{1, i} \sqrt{\beta^{\prime}} y} \sin \left(b_{1, i}\left(2 \nu a_{1, i} \beta+\sqrt{\beta^{\prime}} y\right)+c_{1, i}\right)+h \int \frac{d t}{\beta^{\prime} \gamma^{\prime}}, \\
h=\sum_{s=1}^{n} d_{2, s} e^{\nu\left(a_{2, s}^{2}-b_{2, s}^{2}\right) \beta+a_{2, s} \sqrt{\beta^{\prime}} y} \sin \left(b_{2, s}\left(2 \nu a_{2, s} \beta+\sqrt{\beta^{\prime}} y\right)+c_{2, s}\right)
\end{gathered}
$$

where $a_{1, i}, b_{1, i}, c_{1, i}, d_{1, i}$ and $a_{2, s}, b_{2, s}, c_{2, s}, d_{2, s}$ are real constants.

Case 2. $r=0, b c \neq 0$ or $r=1, b \neq 0$ if $r=1$.

For convenience, we denote

$$
a=\sqrt{4 \delta_{0, r} b c+\delta_{1, r} b^{2}} .
$$


Then we have the following solution of the systems (2.31) and (2.32):

$$
\begin{gathered}
g=\cosh \left(a \int \frac{d t}{\beta^{\prime} \gamma^{\prime}}\right) \sum_{i=1}^{m} d_{1, i} e^{\nu\left(a_{1, i}^{2}-b_{1, i}^{2}\right) \beta+a_{1, i} \sqrt{\beta^{\prime}} y} \sin \left(b_{1, i}\left(2 \nu a_{1, i} \beta+\sqrt{\beta^{\prime}} y\right)+c_{1, i}\right) \\
+\frac{\sinh \left(a \int \frac{d t}{\beta^{\prime} \gamma^{\prime}}\right)}{a} \sum_{s=1}^{n} d_{2, s} e^{\nu\left(a_{2, s}^{2}-b_{2, s}^{2}\right) \beta+a_{2, s} \sqrt{\beta^{\prime}} y} \sin \left(b_{2, s}\left(2 \nu a_{2, s} \beta+\sqrt{\beta^{\prime}} y\right)+c_{2, s}\right), \quad(2.36) \\
h=a \sinh \left(a \int \frac{d t}{\beta^{\prime} \gamma^{\prime}}\right) \sum_{i=1}^{m} d_{1, i} e^{\nu\left(a_{1, i}^{2}-b_{1, i}^{2}\right) \beta+a_{1, i} \sqrt{\beta^{\prime}} y} \sin \left(b_{1, i}\left(2 \nu a_{1, i} \beta+\sqrt{\beta^{\prime}} y\right)+c_{1, i}\right) \\
+\cosh \left(\int \frac{a d t}{\beta^{\prime} \gamma^{\prime}}\right) \sum_{s=1}^{n} d_{2, s} e^{\nu\left(a_{2, s}^{2}-b_{2, s}^{2}\right) \beta+a_{2, s} \sqrt{\beta^{\prime}} y} \sin \left(b_{2, s}\left(2 \nu a_{2, s} \beta+\sqrt{\beta^{\prime}} y\right)+c_{2, s}\right) .
\end{gathered}
$$

In any case,

$$
\begin{aligned}
& \Phi_{1}=\left(\frac{2 \beta^{\prime}{\beta^{\prime \prime}}^{\prime \prime}-\left(\beta^{\prime \prime}\right)^{2}}{4\left(\beta^{\prime}\right)^{2}}+\frac{2 \gamma^{\prime} \gamma^{\prime \prime \prime}-\left(\gamma^{\prime \prime}\right)^{2}}{4\left(\gamma^{\prime}\right)^{2}}+\frac{\beta^{\prime \prime} \gamma^{\prime \prime}}{2 \beta^{\prime} \gamma^{\prime}}+\frac{\delta_{0, r} 4 b c+\delta_{1, r} b^{2}}{\left(\beta^{\prime} \gamma^{\prime}\right)^{2}}\right) x, \\
& \Phi_{3}=\left[\frac{\left(3\left(\gamma^{\prime \prime}\right)^{2}-2 \gamma^{\prime}{\gamma^{\prime \prime}}^{\prime}\right) z^{2}}{8\left(\gamma^{\prime}\right)^{2}}+\frac{\xi_{r}^{2}-(-1)^{r}\left(2 \beta^{\prime \prime} \gamma^{\prime}+5 \beta^{\prime} \gamma^{\prime \prime}\right) \zeta_{r}}{2\left(\beta^{\prime}\right)^{3}\left(\gamma^{\prime}\right)^{3}}\right]_{z} .
\end{aligned}
$$

Thus (2.4), (2.38), (2.39) and the first equation in (2.22) give

$$
\begin{aligned}
p & =\rho\left[\frac{\left(2 \beta^{\prime}{\beta^{\prime \prime}}^{\prime \prime}-3\left({\beta^{\prime \prime}}^{\prime}\right) y^{2}\right.}{8\left(\beta^{\prime}\right)^{2}}+\frac{2 \gamma^{\prime}{\gamma^{\prime \prime}}^{\prime \prime}-3\left(\gamma^{\prime \prime}\right)^{2}}{8\left(\gamma^{\prime}\right)^{2}} z^{2}+\frac{(-1)^{r}\left(2 \beta^{\prime \prime} \gamma^{\prime}+5 \beta^{\prime} \gamma^{\prime \prime}\right) \zeta_{r}-\xi_{r}^{2}}{2\left(\beta^{\prime}\right)^{3}\left(\gamma^{\prime}\right)^{3}}\right] \\
& +\frac{\rho x^{2}}{2}\left(\frac{\left(\beta^{\prime \prime}\right)^{2}-2 \beta^{\prime}{\beta^{\prime \prime}}^{\prime \prime}}{4\left(\beta^{\prime}\right)^{2}}+\frac{\left(\gamma^{\prime \prime}\right)^{2}-2 \gamma^{\prime}{\gamma^{\prime \prime}}^{\prime \prime}}{4\left(\gamma^{\prime}\right)^{2}}-\frac{\beta^{\prime \prime} \gamma^{\prime \prime}}{2 \beta^{\prime} \gamma^{\prime}}-\frac{\delta_{0, r} 4 b c+\delta_{1, r} b^{2}}{\left(\beta^{\prime} \gamma^{\prime}\right)^{2}}\right)
\end{aligned}
$$

modulo the transformation in (1.10).

By (2.20), (2.27), (2.28), (2.33), (2.34), (2.36) and (2.37), we obtain:

Theorem 2.2. Let $\beta, \gamma$ be functions in $t$, and let $b, c, a_{1, i}, b_{1, i}, c_{1, i}, d_{1, i}$ and $a_{2, s}, b_{2, s}, c_{2, s}$, $d_{2, s}$ be real constants. Define $\xi_{r}$ and $\zeta_{r}$ in (2.25) and (2.26). For $r=0,1$, we have the following solution of Navier-Stokes equations (1.1)-(1.4):

$$
v=-\frac{\beta^{\prime \prime}}{2 \beta^{\prime}} y, \quad w=\frac{\xi_{r}}{\beta^{\prime} \sqrt{\left(\gamma^{\prime}\right)^{3}}}-\frac{\gamma^{\prime \prime}}{2 \gamma^{\prime}} z,
$$

$p$ is given in (2.40), and

$$
\begin{aligned}
u & =\frac{\zeta_{r}}{\sqrt{\beta^{\prime} \gamma^{\prime}}} \sum_{i=1}^{m} d_{1, i} e^{\nu\left(a_{1, i}^{2}-b_{1, i}^{2}\right) \beta+a_{1, i} \sqrt{\beta^{\prime}} y} \sin \left(b_{1, i}\left(2 \nu a_{1, i} \beta+\sqrt{\beta^{\prime}} y\right)+c_{1, i}\right) \\
& +\left(\frac{\beta^{\prime \prime}}{2 \beta^{\prime}}+\frac{\gamma^{\prime \prime}}{2 \gamma^{\prime}}-\frac{\zeta_{r}}{\beta^{\prime} \gamma^{\prime}}\right) x+\frac{1+\zeta_{r} \int \frac{d t}{\beta^{\prime} \gamma^{\prime}}}{\sqrt{\beta^{\prime} \gamma^{\prime}}} \sum_{s=1}^{n} d_{2, s} e^{\nu\left(a_{2, s}^{2}-b_{2, s}^{2}\right) \beta+a_{2, s} \sqrt{\beta^{\prime}} y} \\
& \times \sin \left(b_{2, s}\left(2 \nu a_{2, s} \beta+\sqrt{\beta^{\prime}} y\right)+c_{2, s}\right)
\end{aligned}
$$




$$
\text { if } \begin{aligned}
r & =0, b c=0, \text { and } \\
u & =\left(\frac{\beta^{\prime \prime}}{2 \beta^{\prime}}+\frac{\gamma^{\prime \prime}}{2 \gamma^{\prime}}-\frac{\zeta_{r}}{\beta^{\prime} \gamma^{\prime}}\right) x+\frac{1}{\sqrt{\beta^{\prime} \gamma^{\prime}}}\left(\zeta_{r} \cosh \left(\int \frac{a d t}{\beta^{\prime} \gamma^{\prime}}\right)+a \sinh \left(\int \frac{a d t}{\beta^{\prime} \gamma^{\prime}}\right)\right) \\
& \times \sum_{i=1}^{m} d_{1, i} e^{\nu\left(a_{1, i}^{2}-b_{1, i}^{2}\right) \beta+a_{1, i} \sqrt{\beta^{\prime}} y} \sin \left(b_{1, i}\left(2 \nu a_{1, i} \beta+\sqrt{\beta^{\prime}} y\right)+c_{1, i}\right) \\
& +\frac{1}{\sqrt{\beta^{\prime} \gamma^{\prime}}}\left(\cosh \left(\int \frac{a d t}{\beta^{\prime} \gamma^{\prime}}\right)+\frac{\zeta_{r}}{a} \sinh \left(\int \frac{a d t}{\beta^{\prime} \gamma^{\prime}}\right)\right) \sum_{s=1}^{n} d_{2, s} e^{\nu\left(a_{2, s}^{2}-b_{2, s}^{2}\right) \beta+a_{2, s} \sqrt{\beta^{\prime}} y} \\
& \times \sin \left(b_{2, s}\left(2 \nu a_{2, s} \beta+\sqrt{\beta^{\prime}} y\right)+c_{2, s}\right)
\end{aligned}
$$

if $r=0, b c \neq 0$ or $r=1, b \neq 0$, where $a$ is defined in (2.35).

REMARK 2.3. We can use Fourier expansion to solve system (2.31) and (2.32) for $g\left(\beta, \sqrt{\beta^{\prime}} y\right)$ and $h\left(\beta, \sqrt{\beta^{\prime}} y\right)$ with given $g\left(0, \sqrt{\beta^{\prime}} y\right)$ and $h\left(0, \sqrt{\beta^{\prime}} y\right)$. In this way, we can obtain discontinuous solutions of Navier-Stokes equations (1.1)-(1.4), which may be useful in studying shock waves.

Set

$$
\varpi=x^{2}+y^{2}
$$

Consider

$$
u=\frac{\alpha^{\prime}}{2 \alpha} x+y \phi(t, \varpi), \quad v=\frac{\alpha^{\prime}}{2 \alpha} y-x \phi(t, \varpi), \quad w=\psi(t, \varpi)-\frac{\alpha^{\prime}}{\alpha} z,
$$

where $\alpha$ is a function in $t$ and $\phi, \psi$ are functions in $t, \varpi$. Then (2.1)-(2.3) give

$$
\begin{aligned}
\Phi_{1} & =\frac{2 \alpha \alpha^{\prime \prime}-\left(\alpha^{\prime}\right)^{2}}{4 \alpha^{2}} x+y \phi_{t}+\frac{\alpha^{\prime} y}{\alpha}(\varpi \phi)_{\varpi}-x \phi^{2}-4 y \nu(\varpi \phi)_{\varpi \varpi}, \\
\Phi_{2} & =\frac{2 \alpha \alpha^{\prime \prime}-\left(\alpha^{\prime}\right)^{2}}{4 \alpha^{2}} y-x \phi_{t}-\frac{\alpha^{\prime} x}{\alpha}(\varpi \phi)_{\varpi}-y \phi^{2}+4 x \nu(\varpi \phi)_{\varpi \varpi}, \\
\Phi_{3} & =\frac{2\left(\alpha^{\prime}\right)^{2}-\alpha \alpha^{\prime \prime}}{\alpha^{2}} z+\psi_{t}-\frac{\alpha^{\prime}}{\alpha} \psi+\frac{\alpha^{\prime}}{\alpha} \varpi \psi_{\varpi}-4 \nu\left(\psi_{\varpi}+\varpi \psi_{\varpi \varpi}\right) .
\end{aligned}
$$

Note that $\partial_{y}\left(\Phi_{1}\right)=\partial_{x}\left(\Phi_{2}\right)$ becomes

$$
(\varpi \phi)_{\varpi t}+\frac{\alpha^{\prime}}{\alpha}\left((\varpi \phi)_{\varpi}+\varpi(\varpi \phi)_{\varpi \varpi}\right)-4 \nu\left((\varpi \phi)_{\varpi \varpi}+\varpi(\varpi \phi)_{\varpi \varpi \varpi}\right)=0 .
$$

Set

$$
\hat{\phi}=\alpha(\varpi \phi)_{\varpi}
$$

Then (2.49) becomes

$$
\hat{\phi}_{t}+\frac{\alpha^{\prime}}{\alpha} \varpi \hat{\phi}_{\varpi}-4 \nu\left(\hat{\phi}_{\varpi}+\varpi \hat{\phi}_{\varpi \varpi}\right)=0 .
$$

So we have the solution

$$
\hat{\phi}=\sum_{i=0}^{\infty} \frac{\left(\alpha \partial_{t}\right)^{i}(\Im)}{(i !)^{2}}\left(\frac{\varpi}{4 \nu \alpha}\right)^{i}
$$

for a polynomial $\Im$ in $t$. By (2.50)-(2.52), we have

$$
\phi=\beta \varpi^{-1}+\sum_{i=0}^{\infty} \frac{\left(\alpha \partial_{t}\right)^{i}(\Im)}{i !(i+1) ! \alpha}\left(\frac{\varpi}{4 \nu \alpha}\right)^{i}
$$


for a function $\beta$ of $t$.

Note that

$$
\begin{gathered}
\phi_{t}=\beta^{\prime} \varpi^{-1}+\sum_{i=0}^{\infty}\left[\frac{\left(\alpha \partial_{t}\right)^{i+1}(\Im)}{i !(i+1) ! \alpha^{2}}-\frac{\alpha^{\prime}\left(\alpha \partial_{t}\right)^{i}(\Im)}{(i !)^{2} ! \alpha^{2}}\right]\left(\frac{\varpi}{4 \nu \alpha}\right)^{i}, \\
\frac{\alpha^{\prime}}{\alpha}(\varpi \phi)_{\varpi}=\frac{\alpha^{\prime}}{\alpha^{2}} \hat{\phi}=\frac{\alpha^{\prime}}{\alpha^{2}} \sum_{i=0}^{\infty} \frac{\left(\alpha \partial_{t}\right)^{i}(\Im)}{(i !)^{2}}\left(\frac{\varpi}{4 \nu \alpha}\right)^{i}, \\
4 \nu(\varpi \phi)_{\varpi \varpi}=\frac{4 \nu \hat{\phi}_{\varpi}}{\alpha}=\sum_{i=1}^{\infty} \frac{\left(\alpha \partial_{t}\right)^{i}(\Im)}{(i-1) ! i ! \alpha^{2}}\left(\frac{\varpi}{4 \nu \alpha}\right)^{i-1}=\sum_{i=0}^{\infty} \frac{\left(\alpha \partial_{t}\right)^{i+1}(\Im)}{i !(i+1) ! \alpha^{2}}\left(\frac{\varpi}{4 \nu \alpha}\right)^{i} .
\end{gathered}
$$

Thus

$$
\phi_{t}+\frac{\alpha^{\prime}}{\alpha}(\varpi \phi)_{\varpi}-4 \nu(\varpi \phi)_{\varpi \varpi}=\beta^{\prime} \varpi^{-1}
$$

Therefore,

$$
\Phi_{1}=\frac{2 \alpha \alpha^{\prime \prime}-\left(\alpha^{\prime}\right)^{2}}{4 \alpha^{2}} x+\frac{\beta^{\prime} y}{x^{2}+y^{2}}-x \phi^{2}
$$

and

$$
\Phi_{2}=\frac{2 \alpha \alpha^{\prime \prime}-\left(\alpha^{\prime}\right)^{2}}{4 \alpha^{2}} y-\frac{\beta^{\prime} x}{x^{2}+y^{2}}-y \phi^{2} .
$$

On the other hand, the equations $\partial_{z}\left(\Phi_{1}\right)=\partial_{x}\left(\Phi_{3}\right)$ and $\partial_{z}\left(\Phi_{2}\right)=\partial_{y}\left(\Phi_{3}\right)$ are implied by the following differential equation:

$$
\psi_{t}-\frac{\alpha^{\prime}}{\alpha} \psi+\frac{\alpha^{\prime}}{\alpha} \varpi \psi_{\varpi}-4 \nu\left(\psi_{\varpi}+\varpi \psi_{\varpi \varpi}\right)=0
$$

(cf. (2.48)). Similarly, we have the solution

$$
\psi=\alpha \sum_{s=0}^{\infty} \frac{\left(\alpha \partial_{t}\right)^{s}(\varphi)}{(s !)^{2}}\left(\frac{\varpi}{4 \nu \alpha}\right)^{s}
$$

where $\varphi$ is a polynomial in $t$. With this $\psi$,

$$
\Phi_{3}=\frac{2\left(\alpha^{\prime}\right)^{2}-\alpha \alpha^{\prime \prime}}{\alpha^{2}} z
$$

By $(2.4),(2.44),(2.45),(2.53),(2.58),(2.59),(2.61)$ and (2.62), we obtain: 
Theorem 2.4. Let $\alpha, \beta$ be any functions in $t$ and let $\Im, \varphi$ be polynomials in $t$. We have the following solution of the Navier-Stokes equations (1.1)-(1.4):

$$
\begin{gathered}
u=\frac{\alpha^{\prime}}{2 \alpha} x+\frac{\beta y}{x^{2}+y^{2}}+y \sum_{i=0}^{\infty} \frac{\left(\alpha \partial_{t}\right)^{i}(\Im)}{i !(i+1) ! \alpha}\left(\frac{x^{2}+y^{2}}{4 \nu \alpha}\right)^{i}, \\
v=\frac{\alpha^{\prime}}{2 \alpha} y-\frac{\beta x}{x^{2}+y^{2}}-x \sum_{i=0}^{\infty} \frac{\left(\alpha \partial_{t}\right)^{i}(\Im)}{i !(i+1) ! \alpha}\left(\frac{x^{2}+y^{2}}{4 \nu \alpha}\right)^{i}, \\
w=\alpha \sum_{s=0}^{\infty} \frac{\left(\alpha \partial_{t}\right)^{s}(\varphi)}{(s !)^{2}}\left(\frac{x^{2}+y^{2}}{4 \nu \alpha}\right)^{s}-\frac{\alpha^{\prime}}{\alpha} z, \\
p=\frac{\rho\left(\left(\alpha^{\prime}\right)^{2}-2 \alpha \alpha^{\prime \prime}\right)\left(x^{2}+y^{2}\right)}{8 \alpha^{2}}+\frac{\rho\left(\alpha \alpha^{\prime \prime}-2\left(\alpha^{\prime}\right)^{2}\right) z^{2}}{\alpha^{2}}+\beta^{\prime} \arctan \frac{y}{x} \\
+2 \nu \rho \alpha \sum_{i, s=0}^{\infty} \frac{\left[\left(\alpha \partial_{t}\right)^{i}(\Im)\right]\left[\left(\alpha \partial_{t}\right)^{s}(\varphi)\right]}{i !(i+1) ! s !(s+1) !(\alpha)^{2}}\left(\frac{x^{2}+y^{2}}{4 \nu \alpha}\right)^{i+s+1} .
\end{gathered}
$$

REMARK 2.5. The above solution can be used to describe incompressible fluid in a nozzle. The polynomials $\Im$ and $\varphi$ can be replaced by the other functions as long as the related power series converge.

3. Moving-frame approach I. In this section, we will present the general settings for the moving-frame approach and find two families of exact solutions.

Let $\alpha, \beta$ be given functions of $t$. Denote

$$
T=\left(\begin{array}{ccc}
\cos \alpha & \sin \alpha \cos \beta & \sin \alpha \sin \beta \\
-\sin \alpha & \cos \alpha \cos \beta & \cos \alpha \sin \beta \\
0 & -\sin \beta & \cos \beta
\end{array}\right)
$$

and

$$
Q=\left(\begin{array}{ccc}
0 & \alpha^{\prime} & \beta^{\prime} \sin \alpha \\
-\alpha^{\prime} & 0 & \beta^{\prime} \cos \alpha \\
-\beta^{\prime} \sin \alpha & -\beta^{\prime} \cos \alpha & 0
\end{array}\right)
$$

Then

$$
T^{-1}=T^{t}=\left(\begin{array}{ccc}
\cos \alpha & -\sin \alpha & 0 \\
\sin \alpha \cos \beta & \cos \alpha \cos \beta & -\sin \beta \\
\sin \alpha \sin \beta & \cos \alpha \sin \beta & \cos \beta
\end{array}\right)
$$

and

$$
\frac{d}{d t}(T)=Q T .
$$

Define the moving frames

$$
\left(\begin{array}{c}
\mathcal{U} \\
\mathcal{V} \\
\mathcal{W}
\end{array}\right)=T\left(\begin{array}{c}
u \\
v \\
w
\end{array}\right), \quad\left(\begin{array}{l}
\mathcal{X} \\
\mathcal{Y} \\
\mathcal{Z}
\end{array}\right)=T\left(\begin{array}{l}
x \\
y \\
z
\end{array}\right) .
$$


Note that

$$
\begin{gathered}
\Delta=\partial_{x}^{2}+\partial_{y}^{2}+\partial_{z}^{2}=\partial_{\mathcal{X}}^{2}+\partial_{\mathcal{Y}}^{2}+\partial_{\mathcal{Z}}^{2}, \quad u \partial_{x}+v \partial_{y}+z \partial_{z}=\mathcal{U} \partial_{\mathcal{X}}+\mathcal{V} \partial_{\mathcal{Y}}+\mathcal{W} \partial_{\mathcal{Z}} \\
u_{x}+v_{y}+w_{z}=\mathcal{U}_{\mathcal{X}}+\mathcal{V}_{\mathcal{Y}}+\mathcal{W}_{\mathcal{Z}}, \quad\left(\begin{array}{c}
\mathcal{X}_{t} \\
\mathcal{Y}_{t} \\
Z_{t}
\end{array}\right)=Q\left(\begin{array}{c}
\mathcal{X} \\
\mathcal{Y} \\
\mathcal{Z}
\end{array}\right) \\
\left(\begin{array}{c}
\partial_{\mathcal{X}} \\
\partial_{\mathcal{Y}} \\
\partial_{\mathcal{Z}}
\end{array}\right)=T\left(\begin{array}{c}
\partial_{x} \\
\partial_{y} \\
\partial_{z}
\end{array}\right), \quad\left(\begin{array}{c}
\partial_{t}(\mathcal{U}) \\
\partial_{t}(\mathcal{V}) \\
\partial_{t}(\mathcal{W})
\end{array}\right)=Q\left(\begin{array}{c}
\mathcal{U} \\
\mathcal{V} \\
\mathcal{W}
\end{array}\right)+T\left(\begin{array}{c}
u_{t} \\
v_{t} \\
w_{t}
\end{array}\right)
\end{gathered}
$$

Write $\mathcal{U}, \mathcal{V}, \mathcal{W}, p$ as functions in $t, \mathcal{X}, \mathcal{Y}, \mathcal{Z}$. Set

$$
\begin{gathered}
R_{1}=\mathcal{U}_{t}+\alpha^{\prime}\left(\mathcal{Y} \mathcal{U}_{\mathcal{X}}-\mathcal{X} \mathcal{U}_{\mathcal{Y}}-\mathcal{V}\right)+\beta^{\prime}\left(\mathcal{Z} \mathcal{U}_{\mathcal{X}}-\mathcal{X} \mathcal{U}_{\mathcal{Z}}-\mathcal{W}\right) \sin \alpha \\
+\beta^{\prime}\left(\mathcal{Z} \mathcal{U}_{\mathcal{Y}}-\mathcal{Y} \mathcal{U}_{\mathcal{Z}}\right) \cos \alpha+\mathcal{U} \mathcal{U}_{\mathcal{X}}+\mathcal{V} \mathcal{U}_{\mathcal{Y}}+\mathcal{W} \mathcal{U}_{\mathcal{Z}}-\nu \Delta(\mathcal{U}) \\
R_{2}=\mathcal{V}_{t}+\alpha^{\prime}\left(\mathcal{Y} \mathcal{V}_{\mathcal{X}}-\mathcal{X} \mathcal{V}_{\mathcal{Y}}+\mathcal{U}\right)+\beta^{\prime}\left(\mathcal{Z} \mathcal{V}_{\mathcal{X}}-\mathcal{X} \mathcal{V}_{\mathcal{Z}}\right) \sin \alpha \\
+\beta^{\prime}\left(\mathcal{Z} \mathcal{V}_{\mathcal{Y}}-\mathcal{Y} \mathcal{V}_{\mathcal{Z}}-\mathcal{W}\right) \cos \alpha+\mathcal{U} \mathcal{V}_{\mathcal{X}}+\mathcal{V} \mathcal{V}_{\mathcal{Y}}+\mathcal{W} \mathcal{V}_{\mathcal{Z}}-\nu \Delta(\mathcal{V}) \\
R_{3}=\mathcal{W}_{t}+\alpha^{\prime}\left(\mathcal{Y} \mathcal{W}_{\mathcal{X}}-\mathcal{X} \mathcal{W}_{\mathcal{Y}}\right)+\beta^{\prime}\left(\mathcal{Z} \mathcal{W}_{\mathcal{X}}-\mathcal{X} \mathcal{W}_{\mathcal{Z}}+\mathcal{U}\right) \sin \alpha \\
+\beta^{\prime}\left(\mathcal{Z} \mathcal{W}_{\mathcal{Y}}-\mathcal{Y} \mathcal{W}_{\mathcal{Z}}+\mathcal{V}\right) \cos \alpha+\mathcal{U} \mathcal{W}_{\mathcal{X}}+\mathcal{V} \mathcal{W}_{\mathcal{Y}}+\mathcal{W} \mathcal{W}_{\mathcal{Z}}-\nu \Delta(\mathcal{W})
\end{gathered}
$$

Then the Navier-Stokes equations (1.1)-(1.4) become

$$
\begin{aligned}
& R_{1}+\frac{1}{\rho} p_{\mathcal{X}}=0, \quad R_{2}+\frac{1}{\rho} p_{\mathcal{Y}}=0, \quad R_{3}+\frac{1}{\rho} p_{\mathcal{Z}}=0, \\
& \mathcal{U}_{\mathcal{X}}+\mathcal{V}_{\mathcal{Y}}+\mathcal{W}_{\mathcal{Z}}=0
\end{aligned}
$$

Instead of solving the equations in (3.12), we will first solve the following compatibility equations:

$$
\partial_{\mathcal{Y}}\left(R_{1}\right)=\partial_{\mathcal{X}}\left(R_{2}\right), \quad \partial_{\mathcal{Z}}\left(R_{1}\right)=\partial_{\mathcal{X}}\left(R_{3}\right), \quad \partial_{\mathcal{Z}}\left(R_{2}\right)=\partial_{\mathcal{Y}}\left(R_{3}\right)
$$

for $\mathcal{U}, \mathcal{V}, \mathcal{W}$, and then find $p$ from the equations in (3.12).

Let $f$ be a function in $t, \mathcal{Y}, \mathcal{Z}$ such that $\partial_{\mathcal{Y}}^{2}(f)=\partial_{\mathcal{Z}}^{2}(f)=0$, and let $\phi, \psi$ be functions in $t, \mathcal{X}$. Suppose that $\gamma$ is a function of $t$. Assume

$$
\mathcal{U}=f-2 \gamma^{\prime} \mathcal{X}, \quad \mathcal{V}=\phi+\gamma^{\prime} \mathcal{Y}, \quad \mathcal{W}=\psi+\gamma^{\prime} \mathcal{Z} .
$$

Then

$$
\begin{gathered}
R_{1}=f_{t}-2 \gamma^{\prime \prime} \mathcal{X}-\alpha^{\prime}\left(3 \gamma^{\prime} \mathcal{Y}+\mathcal{X} f_{\mathcal{Y}}+\phi\right)-\beta^{\prime}\left(3 \gamma^{\prime} \mathcal{Z}+\mathcal{X} f_{\mathcal{Z}}+\psi\right) \sin \alpha \\
+\beta^{\prime}\left(\mathcal{Z} f_{\mathcal{Y}}-\mathcal{Y} f_{\mathcal{Z}}\right) \cos \alpha-2 \gamma^{\prime}\left(f-2 \gamma^{\prime} \mathcal{X}\right)+f_{\mathcal{Y}}\left(\phi+\gamma^{\prime} \mathcal{Y}\right)+f_{\mathcal{Z}}\left(\psi+\gamma^{\prime} \mathcal{Z}\right), \\
R_{2}=\phi_{t}+\gamma^{\prime \prime} \mathcal{Y}+\alpha^{\prime}\left(\mathcal{Y} \phi_{\mathcal{X}}-3 \gamma^{\prime} \mathcal{X}+f\right)+\beta^{\prime} \mathcal{Z} \phi_{\mathcal{X}} \sin \alpha-\beta^{\prime} \psi \cos \alpha \\
+\left(f-2 \gamma^{\prime} \mathcal{X}\right) \phi_{\mathcal{X}}+\gamma^{\prime} \phi+\left(\gamma^{\prime}\right)^{2} \mathcal{Y}-\nu \phi_{\mathcal{X X}} \\
R_{3}=\psi_{t}+\gamma^{\prime \prime} \mathcal{Z}+\alpha^{\prime} \mathcal{Y} \psi_{\mathcal{X}}+\beta^{\prime}\left(\mathcal{Z} \psi_{\mathcal{X}}-3 \gamma^{\prime} \mathcal{X}+f\right) \sin \alpha-\nu \psi_{\mathcal{X X}} \\
+\beta^{\prime} \phi \cos \alpha+\left(f-2 \gamma^{\prime} \mathcal{X}\right) \psi_{\mathcal{X}}+\gamma^{\prime}\left(\psi+\gamma^{\prime} \mathcal{Z}\right)
\end{gathered}
$$


Now (3.14) becomes

$$
\begin{gathered}
\phi_{t \mathcal{X}}+\left(\alpha^{\prime} \mathcal{Y}+\beta^{\prime} \mathcal{Z} \sin \alpha+f\right) \phi_{\mathcal{X X}}-\beta^{\prime} \psi_{\mathcal{X}} \cos \alpha-2 \gamma^{\prime}\left(\mathcal{X} \phi_{\mathcal{X}}\right)_{\mathcal{X}} \\
+\gamma^{\prime} \phi_{\mathcal{X}}-\nu \phi_{\mathcal{X} \mathcal{X}}=f_{t \mathcal{Y}}-\beta^{\prime} f_{\mathcal{Z}} \cos \alpha-\gamma^{\prime} f_{\mathcal{Y}} \\
\psi_{t \mathcal{X}}+\left(\alpha^{\prime} \mathcal{Y}+\beta^{\prime} \mathcal{Z} \sin \alpha+f\right) \psi_{\mathcal{X X}}-\nu \psi_{\mathcal{X} \mathcal{X}}+\beta^{\prime} \phi_{\mathcal{X}} \cos \alpha \\
-2\left(\gamma^{\prime} \mathcal{X} \psi_{\mathcal{X}}\right)_{\mathcal{X}}+\gamma^{\prime} \psi_{\mathcal{X}}=f_{t \mathcal{Z}}+\beta^{\prime} f_{\mathcal{Y}} \cos \alpha-\gamma^{\prime} f_{\mathcal{Z}} \\
\alpha^{\prime} f_{\mathcal{Z}}+\left(\beta^{\prime} \sin \alpha+f_{\mathcal{Z}}\right) \phi_{\mathcal{X}}=\left(\alpha^{\prime}+f_{\mathcal{Y}}\right) \psi_{\mathcal{X}}+\beta^{\prime} f_{\mathcal{Y}} \sin \alpha
\end{gathered}
$$

By (3.19) and (3.20), we take

$$
f=-\alpha^{\prime} \mathcal{Y}-\beta^{\prime} \mathcal{Z} \sin \alpha
$$

modulo the transformations of type $T_{1 \alpha}$ in (1.8) and (1.9). Note that (3.21) is implied by (3.22). Integrating (3.19) and (3.20), we obtain

$$
\begin{gathered}
\phi_{t}-2 \gamma^{\prime} \mathcal{X} \phi_{\mathcal{X}}+\gamma^{\prime} \phi-\nu \phi_{\mathcal{X} X}-\beta^{\prime} \psi \cos \alpha=\left[\left(\beta^{\prime}\right)^{2} \sin \alpha \cos \alpha+\alpha^{\prime} \gamma^{\prime}-\alpha^{\prime \prime}\right] \mathcal{X}+\beta_{1}, \\
\psi_{t}-2 \gamma^{\prime} \mathcal{X} \psi_{\mathcal{X}}+\gamma^{\prime} \psi-\nu \psi_{\mathcal{X} \mathcal{X}}+\beta^{\prime} \phi \cos \alpha \\
=-\left[\left(\beta^{\prime} \sin \alpha\right)^{\prime}+\alpha^{\prime} \beta^{\prime} \cos \alpha-\gamma^{\prime} \beta^{\prime} \sin \alpha\right] \mathcal{X}+\beta_{2}
\end{gathered}
$$

where $\beta_{1}$ and $\beta_{2}$ are arbitrary functions of $t$. To solve the above problem, we write

$$
\beta^{\prime}=\frac{\varphi^{\prime}}{\cos \alpha}, \quad \gamma=\frac{1}{4} \ln \mu^{\prime}
$$

and set

$$
\begin{gathered}
\left(\begin{array}{c}
\hat{\phi} \\
\hat{\psi}
\end{array}\right)=\sqrt[4]{\mu^{\prime}}\left(\begin{array}{cc}
\cos \varphi & -\sin \varphi \\
\sin \varphi & \cos \varphi
\end{array}\right)\left(\begin{array}{c}
\phi \\
\psi
\end{array}\right) \\
\left(\begin{array}{c}
\gamma_{1} \\
\gamma_{2}
\end{array}\right)=\int \frac{1}{\sqrt[4]{\mu^{\prime}}}\left(\begin{array}{cc}
\cos \varphi & \sin \varphi \\
-\sin \varphi & \cos \varphi
\end{array}\right)\left(\begin{array}{c}
\left(\varphi^{\prime}\right)^{2} \tan \alpha+\frac{\alpha^{\prime} \mu^{\prime \prime}}{4 \mu^{\prime}}-\alpha^{\prime \prime} \\
-\left(\varphi^{\prime} \tan \alpha\right)^{\prime}-\alpha^{\prime} \varphi^{\prime}+\frac{\mu^{\prime \prime} \varphi^{\prime}}{4 \mu^{\prime}} \tan \alpha
\end{array}\right) d t
\end{gathered}
$$

Then (3.23) and (3.24) are equivalent to

$$
\begin{aligned}
& \hat{\phi}_{t}-\frac{\mu^{\prime \prime}}{2 \mu^{\prime}} \mathcal{X} \hat{\phi}_{\mathcal{X}}-\nu \phi_{\mathcal{X X}}=\gamma_{1}^{\prime} \sqrt{\mu^{\prime}} \mathcal{X}+\varphi_{1}^{\prime}, \\
& \hat{\psi}_{t}-\frac{\mu^{\prime \prime}}{2 \mu^{\prime}} \mathcal{X} \hat{\psi}_{\mathcal{X}}-\nu \psi_{\mathcal{X} \mathcal{X}}=\gamma_{2}^{\prime} \sqrt{\mu^{\prime}} \mathcal{X}+\varphi_{2}^{\prime},
\end{aligned}
$$

where $\varphi_{1}$ and $\varphi_{2}$ are arbitrary functions of $t$. Thus

$$
\begin{aligned}
& \hat{\phi}=\gamma_{1} \sqrt{\mu^{\prime}} \mathcal{X}+\varphi_{1}+\sum_{i=1}^{m} d_{1, i} e^{\nu\left(a_{1, i}^{2}-b_{1, i}^{2}\right) \mu+a_{1, i} \sqrt{\mu^{\prime}} \mathcal{X}} \sin \left(b_{1, i}\left(2 a_{1, i} \mu+\sqrt{\mu^{\prime}} \mathcal{X}\right)+c_{i, 1}\right), \\
& \hat{\psi}=\gamma_{2} \sqrt{\mu^{\prime}} \mathcal{X}+\varphi_{2}+\sum_{s=1}^{n} d_{1, s} e^{\nu\left(a_{2, s}^{2}-b_{1, s}^{2}\right) \mu+a_{2, s} \sqrt{\mu^{\prime}} \mathcal{X}} \sin \left(b_{2, s}\left(2 a_{2, s} \mu+\sqrt{\mu^{\prime}} \mathcal{X}\right)+c_{2, s}\right),
\end{aligned}
$$


where $a_{1, i}, a_{2, s}, b_{1, i}, b_{2, s}, c_{1, i}, c_{2, s}, d_{1, i}$ and $d_{2, s}$ are real constants. According (3.26), we have

$$
\begin{gathered}
\phi=\sqrt[4]{\mu^{\prime}}\left(\gamma_{1} \cos \varphi+\gamma_{2} \sin \varphi\right) \mathcal{X}+\sigma_{1}+\frac{\cos \varphi}{\sqrt[4]{\mu^{\prime}}} \sum_{i=1}^{m} d_{1, i} e^{\nu\left(a_{1, i}^{2}-b_{1, i}^{2}\right) \mu+a_{1, i} \sqrt{\mu^{\prime}} \mathcal{X}} \\
\times \sin \left(b_{1, i}\left(2 a_{1, i}+\sqrt{\mu^{\prime}} \mathcal{X}\right)+c_{i, 1}\right)+\frac{\sin \varphi}{\sqrt[4]{\mu^{\prime}}} \sum_{s=1}^{n} d_{1, s} e^{\nu\left(a_{2, s}^{2}-b_{1, s}^{2}\right) \mu+a_{2, s} \sqrt{\mu^{\prime}} \mathcal{X}} \\
\times \sin \left(b_{2, s}\left(2 a_{2, s}+\sqrt{\mu^{\prime}} \mathcal{X}\right)+c_{2, s}\right), \\
\psi \sqrt[4]{\mu^{\prime}}\left(\gamma_{2} \cos \varphi-\gamma_{1} \sin \varphi\right) \mathcal{X}+\sigma_{2}-\frac{\sin \varphi}{\sqrt[4]{\mu^{\prime}}} \sum_{i=1}^{m} d_{1, i} e^{\nu\left(a_{1, i}^{2}-b_{1, i}^{2}\right) \mu+a_{1, i} \sqrt{\mu^{\prime}} \mathcal{X}} \\
\times \sin \left(b_{1, i}\left(2 a_{1, i}+\sqrt{\mu^{\prime}} \mathcal{X}\right)+c_{i, 1}\right)+\frac{\cos \varphi}{\sqrt[4]{\mu^{\prime}}} \sum_{s=1}^{n} d_{1, s} e^{\nu\left(a_{2, s}^{2}-b_{1, s}^{2}\right) \mu+a_{2, s} \sqrt{\mu^{\prime}} \mathcal{X}} \\
\times \sin \left(b_{2, s}\left(2 a_{2, s}+\sqrt{\mu^{\prime}} \mathcal{X}\right)+c_{2, s}\right),
\end{gathered}
$$

where $\sigma_{1}$ and $\sigma_{2}$ are arbitrary functions of $t$.

To find the pressure $p$, we recalculate

$$
\begin{gathered}
R_{1}=\left(\left(\varphi^{\prime}\right)^{2} \mathcal{Y}-2 \varphi^{\prime} \psi\right) \tan \alpha-2 \alpha^{\prime} \phi-\alpha^{\prime \prime} \mathcal{Y}-\left(\varphi^{\prime \prime}+\alpha^{\prime} \varphi^{\prime}\left(1+\sec ^{2} \alpha\right)\right) \mathcal{Z} \\
-\frac{\mu^{\prime \prime}\left(\alpha^{\prime} \mathcal{Y}+\varphi^{\prime} \mathcal{Z} \tan \alpha\right)}{\mu^{\prime}}+\left(\left(\alpha^{\prime}\right)^{2}+\left(\varphi^{\prime}\right)^{2} \tan ^{2} \alpha\right) \mathcal{X}+\frac{\left(3\left(\mu^{\prime \prime}\right)^{2}-2 \mu^{\prime \prime}\right) \mathcal{X}}{4\left(\mu^{\prime}\right)^{2}} \\
R_{2}=\frac{\left(4 \mu^{\prime \prime}-3\left(\mu^{\prime \prime}\right)^{2}\right) \mathcal{Y}}{16\left(\mu^{\prime}\right)^{2}}-\left(\alpha^{\prime}\right)^{2} \mathcal{Y}+\sigma_{1}^{\prime}+\left(\varphi^{\prime} \mathcal{X}-\alpha^{\prime} \mathcal{Z}\right) \varphi^{\prime} \tan \alpha-\frac{\left(\alpha^{\prime} \mu^{\prime \prime}+\alpha^{\prime \prime} \mu^{\prime}\right) \mathcal{X}}{\mu^{\prime}} \\
R_{3}=\frac{\left(4 \mu^{\prime \prime \prime}-3\left(\mu^{\prime \prime}\right)^{2}\right) \mathcal{Z}}{16\left(\mu^{\prime}\right)^{2}}-\frac{\mu^{\prime \prime} \mathcal{X}+\alpha^{\prime} \mu^{\prime} \mathcal{Y}}{\mu^{\prime}} \varphi^{\prime} \tan \alpha+\sigma_{2}^{\prime} \\
-\left(\varphi^{\prime}\right)^{2} \mathcal{Z} \tan ^{2} \alpha-\left(\varphi^{\prime \prime}+\alpha^{\prime} \varphi^{\prime}\left(1+\sec ^{2} \alpha\right)\right) \mathcal{X}
\end{gathered}
$$

Thus

$$
\begin{aligned}
p & =\rho\left\{\left(\alpha^{\prime \prime} \mathcal{Y}+\left(\varphi^{\prime \prime} \tan \alpha+\alpha^{\prime} \varphi^{\prime}\left(1+\sec ^{2} \alpha\right)\right) \mathcal{Z}\right) \mathcal{X}+\frac{\mu^{\prime \prime}\left(\alpha^{\prime} \mathcal{Y}+\varphi^{\prime} \mathcal{Z} \tan \alpha\right) \mathcal{X}}{\mu^{\prime}}\right. \\
& -\sigma_{1}^{\prime} \mathcal{Y}-\sigma_{2}^{\prime} \mathcal{Z}+\frac{\mathcal{X}^{2}}{2}\left(\frac{\left(\mu^{\prime \prime \prime}-\left(3 \mu^{\prime \prime}\right)^{2}\right)}{4\left(\mu^{\prime}\right)^{2}}-\left(\alpha^{\prime}\right)^{2}-\left(\varphi^{\prime}\right)^{2} \tan ^{2} \varphi\right) \\
& +\frac{\left(3\left(\mu^{\prime \prime}\right)^{2}-4 \mu^{\prime \prime \prime}\right)\left(\mathcal{Y}^{2}+\mathcal{Z}^{2}\right)}{32\left(\mu^{\prime}\right)^{2}}+\left(\alpha^{\prime} \mathcal{Z}-\varphi^{\prime} \mathcal{X}\right) \varphi^{\prime} \mathcal{Y} \tan \alpha \\
& \left.+\frac{\left(\alpha^{\prime}\right)^{2} \mathcal{Y}^{2}+\left(\gamma^{\prime}\right)^{2} \mathcal{Z}^{2} \tan ^{2} \alpha}{2}+2 \int\left(\alpha^{\prime} \phi+\varphi^{\prime} \psi \tan \alpha\right) d \mathcal{X}\right\}
\end{aligned}
$$

modulo the transformation in (1.10). In summary, we have: 
Theorem 3.1. Let $\alpha, \varphi, \mu, \sigma_{1}, \sigma_{2}$ be functions of $t$ with $\mu^{\prime}>0$. Take real constants $\left\{a_{1, i}, a_{2, s}, b_{1, i}, b_{2, s}, c_{1, i}, c_{2, s}, d_{1, i}, d_{2, s} \mid i=1, \ldots, m ; s=1, \ldots, n\right\}$. Denote

$$
\beta=\int \frac{\varphi^{\prime} d t}{\cos \alpha}
$$

and define $\gamma_{1}, \gamma_{2}$ by (3.27). Take the notation $\mathcal{X}, \mathcal{Y}, \mathcal{Z}$ given in (3.1) and (3.5). In terms of the functions $\phi$ in (3.32) and $\psi$ in (3.33), we have the following solution of the Navier-Stokes equations (1.1)-(1.4):

$$
\begin{gathered}
u=-\left(\frac{\mu^{\prime \prime} \mathcal{X}}{2 \mu^{\prime}}+\alpha^{\prime} \mathcal{Y}+\varphi^{\prime} \mathcal{Z} \tan \alpha\right) \cos \alpha-\left(\phi+\frac{\mu^{\prime \prime} \mathcal{Y}}{4 \mu^{\prime}}\right) \sin \alpha, \\
v=\left(\frac{\mu^{\prime \prime} \mathcal{X}}{2 \mu^{\prime}}-\alpha^{\prime} \mathcal{Y}-\varphi^{\prime} \mathcal{Z} \tan \alpha\right) \sin \alpha \cos \beta \\
+\left(\phi+\frac{\mu^{\prime \prime} \mathcal{Y}}{4 \mu^{\prime}}\right) \cos \alpha \cos \beta-\left(\psi+\frac{\mu^{\prime \prime} \mathcal{Z}}{4 \mu^{\prime}}\right) \sin \beta, \\
w=\left(\frac{\mu^{\prime \prime} \mathcal{X}}{2 \mu^{\prime}}-\alpha^{\prime} \mathcal{Y}-\varphi^{\prime} \mathcal{Z} \tan \alpha\right) \sin \alpha \sin \beta \\
+\left(\phi+\frac{\mu^{\prime \prime} \mathcal{Y}}{4 \mu^{\prime}}\right) \cos \alpha \sin \beta+\left(\psi+\frac{\mu^{\prime \prime} \mathcal{Z}}{4 \mu^{\prime}}\right) \cos \beta,
\end{gathered}
$$

and $p$ is given in (3.37).

Remark 3.2. We can use Fourier expansion to solve system (3.28) and (3.29) for $\hat{\phi}\left(\mu, \sqrt{\mu^{\prime}} \mathcal{X}\right)$ and $\hat{\psi}\left(\mu, \sqrt{\mu^{\prime}} \mathcal{X}\right)$ with given $\hat{\phi}\left(0, \sqrt{\mu^{\prime}} \mathcal{X}\right)$ and $\hat{\psi}\left(0, \sqrt{\mu^{\prime}} \mathcal{X}\right)$. In this way, we can obtain discontinuous solutions of the Navier-Stokes equations (1.1)-(1.4), which may be useful in studying shock waves.

Let $f, g, h$ be functions of $t, \mathcal{X}, \mathcal{Y}, \mathcal{Z}$ that are linear in $\mathcal{X}, \mathcal{Y}, \mathcal{Z}$ and $f_{\mathcal{X}}+g_{\mathcal{Y}}+h_{\mathcal{Z}}=0$. Based on our experience in [X2], we assume

$$
\mathcal{U}=f-6 \nu \mathcal{X}^{-1}, \quad \mathcal{V}=g-6 \nu \mathcal{Y} \mathcal{X}^{-2}, \quad \mathcal{W}=h .
$$

Then

$$
\begin{gathered}
R_{1}=f_{t}+f f_{\mathcal{X}}+f_{\mathcal{Y}} g+f_{\mathcal{Z}} h-6 \nu f_{\mathcal{X}} \mathcal{X}^{-1}+\alpha^{\prime}\left(\mathcal{Y} f_{\mathcal{X}}-\mathcal{X} f_{\mathcal{Y}}-g\right) \\
+\beta^{\prime}\left(\mathcal{Z} f_{\mathcal{X}}-\mathcal{X} f_{\mathcal{Z}}-h\right) \sin \alpha+\beta^{\prime}\left(\mathcal{Z} f_{\mathcal{Y}}-\mathcal{Y} f_{\mathcal{Z}}\right) \cos \alpha \\
+6 \nu\left(f-\mathcal{Y} f_{\mathcal{Y}}+2 \alpha^{\prime} \mathcal{Y}+\beta^{\prime} \mathcal{Z} \sin \alpha\right) \mathcal{X}^{-2}-24 \nu^{2} \mathcal{X}^{-3} \\
R_{2}=g_{t}+f g_{\mathcal{X}}+g g_{\mathcal{Y}}+g_{\mathcal{Z}} h+\alpha^{\prime}\left(\mathcal{Y} g_{\mathcal{X}}-\mathcal{X}_{g_{\mathcal{Y}}}+f\right)+\beta^{\prime}\left(\mathcal{Z} g_{\mathcal{X}}-\mathcal{X} g_{\mathcal{Z}}\right) \sin \alpha \\
-6 \nu\left(\alpha^{\prime}+g_{\mathcal{X}}\right) \mathcal{X}^{-1}-6 \nu\left(g+\beta^{\prime} \mathcal{Z} \cos \alpha-\alpha^{\prime} \mathcal{X}+\mathcal{Y} g_{\mathcal{Y}}\right) \mathcal{X}^{-2} \\
+\beta^{\prime}\left(\mathcal{Z} g_{\mathcal{Y}}-g_{\mathcal{Z}} \mathcal{Y}-h\right) \cos \alpha+12 \nu \mathcal{Y}\left(f+\alpha^{\prime} \mathcal{Y}+\beta^{\prime} \mathcal{Z} \sin \alpha\right) \mathcal{X}^{-3} \\
R_{3}=h_{t}+f h_{\mathcal{X}}+g h_{\mathcal{Y}}+h h_{\mathcal{Z}}+\alpha^{\prime}\left(\mathcal{Y} h_{\mathcal{X}}-\mathcal{X} h_{\mathcal{Y}}\right)+\beta^{\prime}\left(\mathcal{Z} h_{\mathcal{X}}-\mathcal{X} h_{\mathcal{Z}}+f\right) \sin \alpha \\
+\beta^{\prime}\left(\mathcal{Z} h_{\mathcal{Y}}-\mathcal{Y} h_{\mathcal{Z}}+g\right) \cos \alpha-6 \nu\left(h_{x}+\beta^{\prime} \sin \alpha\right) \mathcal{X}^{-1}-6 \nu\left(h_{\mathcal{Y}}+\beta^{\prime} \cos \alpha\right) \mathcal{Y} \mathcal{X}^{-2} .
\end{gathered}
$$

According to the negative powers of $\mathcal{X}$ in (3.14), we take

$$
\begin{gathered}
f=\gamma \mathcal{X}-\alpha^{\prime} \mathcal{Y}-\beta^{\prime} \mathcal{Z} \sin \alpha, \quad g=\alpha^{\prime} \mathcal{X}+\gamma \mathcal{Y}-\beta^{\prime} \mathcal{Z} \cos \alpha \\
h=-\left(\beta^{\prime} \mathcal{X} \sin \alpha+\beta^{\prime} \mathcal{Y} \cos \alpha+2 \gamma \mathcal{Z}\right)
\end{gathered}
$$


modulo the transformations of the type in (1.8) and (1.9) for some function $\gamma$ of $t$. With the above data, we have

$$
\begin{gathered}
R_{1}=\left(\gamma^{\prime}+\gamma^{2}-\left(\alpha^{\prime}\right)^{2}+3\left(\beta^{\prime}\right)^{2} \sin ^{2} \alpha\right) \mathcal{X}+12 \nu \alpha^{\prime} \mathcal{Y} \mathcal{X}^{-2}-24 \nu^{2} \mathcal{X}^{-3} \\
+\left(3\left(\beta^{\prime}\right)^{2} \sin \alpha \cos \alpha-\alpha^{\prime \prime}-2 \alpha^{\prime} \gamma\right) \mathcal{Y}+\left(4 \beta^{\prime} \gamma-\beta^{\prime \prime}\right) \mathcal{Z} \sin \alpha, \\
R_{2}=\left(\gamma^{\prime}+\gamma^{2}-\left(\alpha^{\prime}\right)^{2}+3\left(\beta^{\prime}\right)^{2} \cos ^{2} \alpha\right) \mathcal{Y}-12 \nu \alpha^{\prime} \mathcal{X}^{-1} \\
+\left(\alpha^{\prime \prime}+2 \alpha^{\prime} \gamma+3\left(\beta^{\prime}\right)^{2} \sin \alpha \cos \alpha\right) \mathcal{X}+\left(4 \beta^{\prime} \gamma-\beta^{\prime \prime}\right) \mathcal{Z} \cos \alpha, \\
R_{3}=\left(4 \gamma^{2}-2 \gamma^{\prime}-\left(\beta^{\prime}\right)^{2}\right) \mathcal{Z}+\left(4 \beta^{\prime} \gamma-\beta^{\prime \prime}\right)(\mathcal{X} \sin \alpha+\mathcal{Y} \cos \alpha) .
\end{gathered}
$$

By (3.48)-(3.50), (3.14) is now equivalent to

$$
-\alpha^{\prime \prime}-2 \alpha^{\prime} \gamma=\alpha^{\prime \prime}+2 \alpha^{\prime} \gamma \Longrightarrow \gamma=-\frac{\alpha^{\prime \prime}}{2 \alpha^{\prime}} .
$$

Thus

$$
\begin{gathered}
\mathcal{U}=-\frac{\alpha^{\prime \prime}}{2 \alpha^{\prime}} \mathcal{X}-\alpha^{\prime} \mathcal{Y}-\beta^{\prime} \mathcal{Z} \sin \alpha-6 \nu \mathcal{X}^{-1}, \\
\mathcal{V}=\alpha^{\prime} \mathcal{X}-\frac{\alpha^{\prime \prime}}{2 \alpha^{\prime}} \mathcal{Y}-\beta^{\prime} \mathcal{Z} \cos \alpha-6 \nu \mathcal{Y} \mathcal{X}^{-2}, \\
\mathcal{W}=\frac{\alpha^{\prime \prime}}{\alpha^{\prime}} \mathcal{Z}-\beta^{\prime} \mathcal{X} \sin \alpha-\beta^{\prime} \mathcal{Y} \cos \alpha
\end{gathered}
$$

by (3.42), (3.46), (3.47) and (3.51). Moreover, (3.12) and (3.48)-(3.50) imply

$$
\begin{aligned}
p & =\rho\left\{\frac{\left(2 \alpha^{\prime} \alpha^{\prime \prime}+4\left(\alpha^{\prime}\right)^{4}-3\left(\alpha^{\prime \prime}\right)^{2}\right)\left(\mathcal{X}^{2}+\mathcal{Y}^{2}\right)}{8\left(\alpha^{\prime}\right)^{2}}-\frac{3\left(\beta^{\prime}\right)^{2}\left(\mathcal{X}^{2} \sin ^{2} \alpha+\mathcal{Y}^{2} \cos ^{2} \alpha\right)}{2}\right. \\
& +12 \nu\left(\alpha^{\prime} \mathcal{Y} \mathcal{X}^{-1}-\nu \mathcal{X}^{-2}\right)+\left({\beta^{\prime \prime}}^{\prime}-4 \beta^{\prime} \gamma\right) \mathcal{Z}(\mathcal{X} \sin \alpha+\mathcal{Y} \cos \alpha) \\
& \left.-3\left(\beta^{\prime}\right)^{2} \mathcal{X Y} \sin \alpha \cos \alpha+\frac{\left(\alpha^{\prime} \alpha^{\prime \prime}+\left(\alpha^{\prime}\right)^{2}\left(\beta^{\prime}\right)^{2}-2\left(\alpha^{\prime \prime}\right)^{2}\right) \mathcal{Z}^{2}}{2\left(\alpha^{\prime}\right)^{2}}\right\} .
\end{aligned}
$$

By (3.3) and (3.5), we have the following theorem:

TheOREm 3.3. Let $\alpha$ and $\beta$ be functions of $t$ with $\alpha^{\prime} \neq 0$. In terms of the notation $\mathcal{X}, \mathcal{Y}, \mathcal{Z}$ given in (3.1) and (3.5), we have the following solution of the Navier-Stokes equations (1.1)-(1.4):

$$
\begin{gathered}
u=\left(\frac{\alpha^{\prime \prime}}{2 \alpha^{\prime}}+6 \nu \mathcal{Y} \mathcal{X}^{-2}\right)(\mathcal{Y} \sin \alpha-\mathcal{X} \cos \alpha)-\alpha^{\prime}(\mathcal{X} \sin \alpha+\mathcal{Y} \cos \alpha) \\
v=-\left(\frac{\alpha^{\prime \prime}}{2 \alpha^{\prime}}+6 \nu \mathcal{Y} \mathcal{X}^{-2}\right)(\mathcal{X} \sin \alpha+\mathcal{Y} \cos \alpha) \cos \beta+\alpha^{\prime}(\mathcal{X} \cos \alpha-\mathcal{Y} \sin \alpha) \cos \beta \\
-\beta^{\prime} \mathcal{Z} \cos \beta+\left(\beta^{\prime} \mathcal{X} \sin \alpha+\beta^{\prime} \mathcal{Y} \cos \alpha-\frac{\alpha^{\prime \prime}}{\alpha^{\prime}} \mathcal{Z}\right) \sin \beta, \\
w=-\left(\frac{\alpha^{\prime \prime}}{2 \alpha^{\prime}}+6 \nu \mathcal{Y} \mathcal{X}^{-2}\right)(\mathcal{X} \sin \alpha+\mathcal{Y} \cos \alpha) \sin \beta+\alpha^{\prime}(\mathcal{X} \cos \alpha-\mathcal{Y} \sin \alpha) \sin \beta \\
-\beta^{\prime} \mathcal{Z} \sin \beta+\left(\frac{\alpha^{\prime \prime}}{\alpha^{\prime}} \mathcal{Z}-\beta^{\prime} \mathcal{X} \sin \alpha-\beta^{\prime} \mathcal{Y} \cos \alpha\right) \cos \beta,
\end{gathered}
$$


and $p$ is given in (3.55). The above solution blows up at any point on the following rotating plane with a line deleted:

$$
\begin{aligned}
& \left\{(x, y, z) \in \mathbb{R}^{3} \mid x \cos \alpha+y \sin \alpha \cos \beta+z \sin \alpha \sin \beta=0\right. \\
& -x \sin \alpha+y \cos \alpha \cos \beta+z \cos \alpha \sin \beta \neq 0\} .
\end{aligned}
$$

We remark that the above solution may be applied to study turbulence.

4. Moving-frame approach II. Motivated from the solution $\psi$ in (2.27) of equation (2.24), we will solve the Navier-Stokes equations by ansatzes with given irrational functions under the moving frames in (3.5).

First we rewrite (3.9)-(3.11):

$$
\begin{gathered}
R_{1}=\mathcal{U}_{t}+\left(\alpha^{\prime} \mathcal{Y}+\beta^{\prime} \mathcal{Z} \sin \alpha+\mathcal{U}\right) \mathcal{U}_{\mathcal{X}}+\left(\mathcal{V}-\alpha^{\prime} \mathcal{X}+\beta^{\prime} \mathcal{Z} \cos \alpha\right) \mathcal{U}_{\mathcal{Y}} \\
+\left(\mathcal{W}-\beta^{\prime}(\mathcal{X} \sin \alpha+\mathcal{Y} \cos \alpha)\right) \mathcal{U}_{\mathcal{Z}}-\alpha^{\prime} \mathcal{V}-\beta^{\prime} \mathcal{W} \sin \alpha-\nu \Delta(\mathcal{U}) \\
R_{2}=\mathcal{V}_{t}+\left(\alpha^{\prime} \mathcal{Y}+\beta^{\prime} \mathcal{Z} \sin \alpha+\mathcal{U}\right) \mathcal{V}_{\mathcal{X}}+\left(\mathcal{V}-\alpha^{\prime} \mathcal{X}+\beta^{\prime} \mathcal{Z} \cos \alpha\right) \mathcal{V}_{\mathcal{Y}} \\
+\left(\mathcal{W}-\beta^{\prime}(\mathcal{X} \sin \alpha+\mathcal{Y} \cos \alpha)\right) \mathcal{V}_{\mathcal{Z}}+\alpha^{\prime} \mathcal{U}-\beta^{\prime} \mathcal{W} \cos \alpha-\nu \Delta(\mathcal{V}) \\
R_{3}=\mathcal{W}_{t}+\left(\alpha^{\prime} \mathcal{Y}+\beta^{\prime} \mathcal{Z} \sin \alpha+\mathcal{U}\right) \mathcal{W}_{\mathcal{X}}+\left(\mathcal{V}-\alpha^{\prime} \mathcal{X}+\beta^{\prime} \mathcal{Z} \cos \alpha\right) \mathcal{W}_{\mathcal{Y}} \\
+\left(\mathcal{W}-\beta^{\prime}(\mathcal{X} \sin \alpha+\mathcal{Y} \cos \alpha)\right) \mathcal{W}_{\mathcal{Z}}+\beta^{\prime}(\mathcal{U} \sin \alpha+\mathcal{V} \cos \alpha)-\nu \Delta(\mathcal{W})
\end{gathered}
$$

Let $\alpha_{1}, \beta_{1}, \gamma$ be functions of $t$, and let $a, b$ be real numbers. Set

$$
\begin{aligned}
\xi_{0}=e^{\alpha_{1} \mathcal{Y}+\beta_{1} \mathcal{Z}}-a e^{-\alpha_{1} \mathcal{Y}-\beta_{1} \mathcal{Z}}, & \zeta_{0}=e^{\alpha_{1} \mathcal{Y}+\beta_{1} \mathcal{Z}}+a e^{-\alpha_{1} \mathcal{Y}-\beta_{1} \mathcal{Z}} \\
\xi_{1}=\sin \left(\alpha_{1} \mathcal{Y}+\beta_{1} \mathcal{Z}\right), & \zeta_{1}=\cos \left(\alpha_{1} \mathcal{Y}+\beta_{1} \mathcal{Z}\right) \\
\phi_{0}=e^{\gamma \mathcal{X}}-b e^{-\gamma \mathcal{X}}, & \zeta_{0}=e^{\gamma \mathcal{X}}+b e^{-\gamma \mathcal{X}} \\
\phi_{1}=\sin (\gamma \mathcal{X}), \quad \psi_{1}=\cos (\gamma \mathcal{X}), & \Delta_{1}=\partial_{\mathcal{Y}}^{2}+\partial_{\mathcal{Z}}^{2}
\end{aligned}
$$

Suppose that $f$ and $h$ are functions in $t, \mathcal{Y}, \mathcal{Z}$. According to (3.46) and (3.47), we assume

$$
\begin{gathered}
\mathcal{U}=-\alpha^{\prime} \mathcal{Y}-\beta^{\prime} \mathcal{Z} \sin \alpha-\left(f \mathcal{Y}+h_{\mathcal{Z}}\right) \mathcal{X}-\left(\alpha_{1} \sigma+\beta_{1} \tau\right) \zeta_{r} \phi_{s} \\
\mathcal{V}=\alpha^{\prime} \mathcal{X}-\beta^{\prime} \mathcal{Z} \cos \alpha+f+\sigma \gamma \xi_{r} \psi_{s}, \quad \mathcal{W}=\beta^{\prime}(\mathcal{X} \sin \alpha+\mathcal{Y} \cos \alpha)+h+\tau \gamma \xi_{r} \psi_{s}
\end{gathered}
$$


By (4.1)-(4.3), we have

$$
\begin{aligned}
& R_{1}=-\left(\alpha_{1} \sigma+\beta_{1} \tau\right)^{\prime} \zeta_{r} \phi_{s}-\left(\alpha_{1} \sigma+\beta_{1} \tau\right)\left[(-1)^{r}\left(\alpha_{1}^{\prime} \mathcal{Y}+\beta_{1}^{\prime} \mathcal{Z}\right) \xi_{r} \phi_{s}+\gamma^{\prime} \mathcal{X} \zeta_{r} \psi_{s}\right] \\
& -\left(f_{\mathcal{Y} t}+h_{\mathcal{Z} t}\right) \mathcal{X}+\left(\left(f_{\mathcal{Y}}+h_{\mathcal{Z}}\right) \mathcal{X}+\left(\alpha_{1} \sigma+\beta_{1} \tau\right) \zeta_{r} \phi_{s}\right)\left(f_{\mathcal{Y}}+h_{\mathcal{Z}}+\gamma\left(\alpha_{1} \sigma+\beta_{1} \tau\right) \zeta_{r} \psi_{s}\right) \\
& -\alpha^{\prime}\left(f+\alpha^{\prime} \mathcal{X}-\beta^{\prime} \mathcal{Z} \cos \alpha+\gamma \sigma \xi_{r} \psi_{s}\right)-\beta^{\prime}\left(\beta^{\prime}(\mathcal{X} \sin \alpha+\mathcal{Y} \cos \alpha)+h+\gamma \tau \xi_{r} \psi_{s}\right) \sin \alpha \\
& -\left(f+\gamma \sigma \xi_{r} \psi_{s}\right)\left(\alpha^{\prime}+\left(f_{\mathcal{Y} \mathcal{Y}}+h_{\mathcal{Y Z}}\right) \mathcal{X}+(-1)^{r} \alpha_{1}\left(\alpha_{1} \sigma+\beta_{1} \tau\right) \xi_{r} \phi_{s}\right)-\left(h+\gamma \tau \xi_{r} \psi_{s}\right) \\
& \times\left(\beta^{\prime} \sin \alpha+\left(f_{\mathcal{Y Z}}+h_{\mathcal{Z Z}}\right) \mathcal{X}+(-1)^{r} \beta_{1}\left(\alpha_{1} \sigma+\beta_{1} \tau\right) \xi_{r} \phi_{s}\right)+\nu\left\{\Delta_{1}\left(f_{\mathcal{Y}}+h_{\mathcal{Z}}\right) \mathcal{X}\right. \\
& \left.+\left(\alpha_{1} \sigma+\beta_{1} \tau\right)\left[(-1)^{r}\left(\alpha_{1}^{2}+\beta_{1}^{2}\right)+(-1)^{s} \gamma^{2}\right] \zeta_{r} \phi_{s}\right\}-\alpha^{\prime \prime} \mathcal{Y}-\left(\beta^{\prime} \sin \alpha\right)^{\prime} \mathcal{Z} \\
& =\left\{\left(\gamma\left(f_{\mathcal{Y}}+h_{\mathcal{Z}}\right)-\gamma^{\prime}\right) \mathcal{X} \zeta_{r} \psi_{s}-(-1)^{r}\left(\alpha_{1}^{\prime} \mathcal{Y}+\beta_{1}^{\prime} \mathcal{Z}+\alpha_{1} f+\beta_{1} h\right) \xi_{r} \phi_{s}\right\}\left(\alpha_{1} \sigma+\beta_{1} \tau\right) \\
& +\left\{\left(\alpha_{1} \sigma+\beta_{1} \tau\right)\left[(-1)^{s} \nu \gamma^{2}+(-1)^{r} \nu\left(\alpha_{1}^{2}+\beta_{1}^{2}\right)+f_{\mathcal{Y}}+h_{\mathcal{Z}}\right]-\left(\alpha_{1} \sigma+\tau \beta_{1}\right)^{\prime}\right\} \zeta_{r} \phi_{s} \\
& -\gamma\left\{2\left(\sigma \alpha^{\prime}+\tau \beta^{\prime} \sin \alpha\right)+\left[\sigma\left(f_{\mathcal{Y} \mathcal{Y}}+h_{\mathcal{Y Z}}\right)+\tau\left(f_{\mathcal{Y Z}}+h_{\mathcal{Z Z}}\right)\right] \mathcal{X}\right\} \xi_{r} \psi_{s}-\left(f_{\mathcal{Y} t}+h_{\mathcal{Z} t}\right) \mathcal{X} \\
& +\left(f_{\mathcal{Y}}+h_{\mathcal{Z}}\right)^{2} \mathcal{X}-f\left(\alpha^{\prime}+\left(f_{\mathcal{Y Y}}+h_{\mathcal{Y Z}}\right) \mathcal{X}\right)-h\left(\beta^{\prime} \sin \alpha+\left(f_{\mathcal{Y Z}}+h_{\mathcal{Z} \mathcal{Z}}\right) \mathcal{X}\right) \\
& -\alpha^{\prime}\left(f+\alpha^{\prime} \mathcal{X}-\beta^{\prime} \mathcal{Z} \cos \alpha\right)-\beta^{\prime}\left(\beta^{\prime}(\mathcal{X} \sin \alpha+\mathcal{Y} \cos \alpha)+h\right) \sin \alpha-\alpha^{\prime \prime} \mathcal{Y} \\
& -\left(\beta^{\prime} \sin \alpha\right)^{\prime} \mathcal{Z}+\nu \Delta_{1}\left(f_{\mathcal{Y}}+h_{\mathcal{Z}}\right) \mathcal{X}+\gamma\left(\alpha_{1} \sigma+\beta_{1} \tau\right)^{2}\left(\delta_{r, 1}+4 a \delta_{r, 0}\right) \phi_{s} \psi_{s}
\end{aligned}
$$

$$
\begin{aligned}
R_{2} & =\alpha^{\prime \prime} \mathcal{X}-\left(\beta^{\prime} \cos \alpha\right)^{\prime} \mathcal{Z}+f_{t}+(\gamma \sigma)^{\prime} \xi_{r} \psi_{s}+\gamma \sigma\left(\left(\alpha_{1}^{\prime} \mathcal{Y}+\beta_{1}^{\prime} \mathcal{Z}\right) \zeta_{r} \psi_{s}+(-1)^{s} \gamma^{\prime} \mathcal{X} \xi_{r} \phi_{s}\right) \\
& -\alpha^{\prime}\left[\alpha^{\prime} \mathcal{Y}+\beta^{\prime} \mathcal{Z} \sin \alpha+\left(f_{\mathcal{Y}}+h_{\mathcal{Z}}\right) \mathcal{X}+\left(\alpha_{1} \sigma+\beta_{1} \tau\right) \zeta_{r} \phi_{s}\right]-\beta^{\prime}\left[\beta^{\prime}(\mathcal{X} \sin \alpha+\mathcal{Y} \cos \alpha)\right. \\
& \left.+h+\gamma \tau \xi_{r} \psi_{s}\right] \cos \alpha-\left[\left(f_{\mathcal{Y}}+h_{\mathcal{Z}}\right) \mathcal{X}+\left(\alpha_{1} \sigma+\beta_{1} \tau\right) \zeta_{r} \phi_{s}\right]\left(\alpha^{\prime}+(-1)^{s} \gamma^{2} \sigma \xi_{r} \phi_{s}\right) \\
& +\left(f+\gamma \sigma \xi_{r} \psi_{s}\right)\left(f_{\mathcal{Y}}+\alpha_{1} \gamma \sigma \zeta_{r} \psi_{s}\right)+\left(h+\gamma \tau \xi_{r} \psi_{s}\right)\left(f_{\mathcal{Z}}-\beta^{\prime} \cos \alpha+\beta_{1} \gamma \sigma \zeta_{r} \psi_{s}\right) \\
& -\nu\left[\Delta_{1}(f)+\gamma \sigma\left((-1)^{r}\left(\alpha_{1}^{2}+\beta_{1}^{2}\right)+(-1)^{s} \gamma^{2}\right)\right] \xi_{r} \psi_{s} \\
& =\alpha^{\prime \prime} \mathcal{X}+f_{t}+\gamma \sigma\left(\alpha_{1}^{\prime} \mathcal{Y}+\beta_{1}^{\prime} \mathcal{Z}+\alpha_{1} f+\beta_{1} h\right) \zeta_{r} \psi_{s}+(-1)^{s} \gamma \sigma\left(\gamma^{\prime}-\gamma\left(f_{\mathcal{Y}}+h_{\mathcal{Z}}\right)\right) \mathcal{X} \xi_{r} \phi_{s} \\
& +\left\{\gamma\left[\sigma f_{\mathcal{Y}}+\tau f_{\mathcal{Z}}-\nu \sigma\left[(-1)^{r}\left(\alpha_{1}^{2}+\beta_{1}^{2}\right)+(-1)^{s} \gamma^{2}\right]-2 \tau \beta^{\prime} \cos \alpha\right]+(\gamma \sigma)^{\prime}\right\} \xi_{r} \psi_{s} \\
& -2 \alpha^{\prime}\left(\alpha_{1} \sigma+\beta_{1} \tau\right) \zeta_{r} \phi_{s}-\left(\beta^{\prime} \cos \alpha\right)^{\prime} \mathcal{Z}-\alpha^{\prime}\left[\alpha^{\prime} \mathcal{Y}+\beta^{\prime} \mathcal{Z} \sin \alpha+2\left(f \mathcal{Y}+h_{\mathcal{Z}}\right) \mathcal{X}\right] \\
& -\beta^{\prime}\left[\beta^{\prime}(\mathcal{X} \sin \alpha+\mathcal{Y} \cos \alpha)+h\right] \cos \alpha+f f_{\mathcal{Y}}+h\left(f_{\mathcal{Z}}-\beta^{\prime} \cos \alpha\right)-\nu \Delta_{1}(f) \\
& +\gamma^{2} \sigma\left(\alpha_{1} \sigma+\beta_{1} \tau\right)\left(4 b \delta_{s, 0}+\delta_{s, 1}\right) \xi_{r} \zeta_{r},
\end{aligned}
$$




$$
\begin{aligned}
R_{3} & =\left(\beta^{\prime} \sin \alpha\right)^{\prime} \mathcal{X}+\left(\beta^{\prime} \cos \alpha\right)^{\prime} \mathcal{Y}+h_{t}+(\tau \gamma)^{\prime} \xi_{r} \psi_{s}+\tau \gamma\left[\left(\alpha_{1}^{\prime} \mathcal{Y}+\beta_{1}^{\prime} \mathcal{Z}\right) \zeta_{r} \psi_{s}\right. \\
& \left.+(-1)^{s} \gamma^{\prime} \mathcal{X} \xi_{r} \phi_{s}\right]-\left[\left(f \mathcal{Y}+h_{\mathcal{Z}}\right) \mathcal{X}+\left(\alpha_{1} \sigma+\beta_{1} \tau\right) \zeta_{r} \phi_{s}\right]\left(\beta^{\prime} \sin \alpha+(-1)^{s} \tau \gamma^{2} \xi_{r} \phi_{s}\right) \\
& +\left(f+\sigma \gamma \xi_{r} \psi_{s}\right)\left(\beta^{\prime} \cos \alpha+h_{\mathcal{Y}}+\alpha_{1} \tau \gamma \zeta_{r} \psi_{s}\right)+\left(h+\tau \gamma \xi_{r} \psi_{s}\right)\left(h_{\mathcal{Z}}+\beta_{1} \tau \gamma \zeta_{r} \psi_{s}\right) \\
& -\beta^{\prime}\left(\alpha^{\prime} \mathcal{Y}+\beta^{\prime} \mathcal{Z} \sin \alpha+\left(f_{\mathcal{Y}}+h_{\mathcal{Z}}\right) \mathcal{X}+\left(\alpha_{1} \sigma+\beta_{1} \tau\right) \zeta_{r} \phi_{s}\right) \sin \alpha+\beta^{\prime}\left(\alpha^{\prime} \mathcal{X}-\beta^{\prime} \mathcal{Z} \cos \alpha\right. \\
& \left.+f+\sigma \gamma \xi_{r} \psi_{s}\right) \cos \alpha-\nu\left[\Delta_{1}(h)+\gamma \tau\left((-1)^{r}\left(\alpha_{1}^{2}+\beta_{1}^{2}\right)+(-1)^{s} \gamma^{2}\right)\right] \xi_{r} \psi_{s} \\
& =\gamma \tau\left(\alpha_{1}^{\prime} \mathcal{Y}+\beta_{1}^{\prime} \mathcal{Z}+\alpha_{1} f+\beta_{1} h\right) \zeta_{r} \psi_{s}+\left\{(\tau \gamma)^{\prime}-\nu \gamma \tau\left[(-1)^{r}\left(\alpha_{1}^{2}+\beta_{1}^{2}\right)+(-1)^{s} \gamma^{2}\right]\right. \\
& \left.+\gamma\left(2 \beta^{\prime} \sigma \cos \alpha+\sigma h_{\mathcal{Y}}+\tau h_{\mathcal{Z}}\right)\right\} \xi_{r} \psi_{s}-2 \beta^{\prime}\left(\alpha_{1} \sigma+\beta_{1} \tau\right) \zeta_{r} \phi_{s} \sin \alpha+(-1)^{s} \gamma \tau\left(\gamma^{\prime}\right. \\
& \left.-\gamma\left(f \mathcal{Y}+h_{\mathcal{Z}}\right)\right) \mathcal{X} \xi_{r} \phi_{s}+\left(\beta^{\prime} \sin \alpha\right)^{\prime} \mathcal{X}+\left(\beta^{\prime} \cos \alpha\right)^{\prime} \mathcal{Y}+h_{t}-\beta^{\prime}\left(f \mathcal{Y}+h_{\mathcal{Z}}\right) \mathcal{X} \sin \alpha \\
& +f\left(\beta^{\prime} \cos \alpha+h_{\mathcal{Y}}\right)+h h_{\mathcal{Z}}-\beta^{\prime}\left(\alpha^{\prime} \mathcal{Y}+\beta^{\prime} \mathcal{Z} \sin \alpha+\left(f_{\mathcal{Y}}+h_{\mathcal{Z}}\right) \mathcal{X}\right) \sin \alpha+\beta^{\prime}\left(\alpha^{\prime} \mathcal{X}\right. \\
& \left.-\beta^{\prime} \mathcal{Z} \cos \alpha+f\right) \cos \alpha-\nu \Delta_{1}(h)+\gamma^{2} \tau\left(\alpha_{1} \sigma+\beta_{1} \tau\right)\left(4 b \delta_{s, 0}+\delta_{s, 1}\right) \xi_{r} \zeta_{r} .
\end{aligned}
$$

By the coefficients of $\xi_{r} \psi_{s}$ in the equation $\partial_{\mathcal{Y}}\left(R_{1}\right)=\partial_{\mathcal{X}}\left(R_{2}\right)$, we have

$$
\gamma^{2} \sigma=(-1)^{r+s+1} \alpha_{1}\left(\alpha_{1} \sigma+\beta_{1} \tau\right), \quad\left[\sigma\left(f_{\mathcal{Y} \mathcal{Y}}+h_{\mathcal{Y Z}}\right)+\tau\left(f_{\mathcal{Y Z}}+h_{\mathcal{Z Z Z}}\right)\right]_{\mathcal{Y}}=0
$$

Moreover, the coefficients of $\zeta_{r} \phi_{s}$ in the equation $\partial_{\mathcal{Y}}\left(R_{1}\right)=\partial_{\mathcal{X}}\left(R_{2}\right)$ suggest

$$
\left(f_{\mathcal{Y}}+h_{\mathcal{Z}}\right) \mathcal{y}=0
$$

which implies the second equation in (4.13). According the coefficients of $\xi_{r} \phi_{s}$ in the equation $\partial_{\mathcal{Y}}\left(R_{1}\right)=\partial_{\mathcal{X}}\left(R_{2}\right)$, we get

$$
\sigma \beta_{1} h_{\mathcal{Y}}=\alpha_{1}\left(\tau f_{\mathcal{Z}}-2 \tau \beta^{\prime} \cos \alpha\right)
$$

Furthermore, the coefficients of $\zeta_{r} \psi_{s}$ in the equation $\partial_{\mathcal{Y}}\left(R_{1}\right)=\partial_{\mathcal{X}}\left(R_{2}\right)$ yield

$$
\alpha_{1} \beta^{\prime} \sin \alpha=\alpha^{\prime} \beta_{1} \text {. }
$$

Symmetrically, we have (4.16),

$$
\gamma^{2} \tau=(-1)^{r+s+1} \beta_{1}\left(\alpha_{1} \sigma+\beta_{1} \tau\right), \quad\left(f_{\mathcal{Y}}+h_{\mathcal{Z}}\right)_{\mathcal{Z}}=0
$$

and

$$
f_{\mathcal{Z}}=h_{\mathcal{Y}}+2 \beta^{\prime} \cos \alpha .
$$

By the first equation in (4.13) and (4.17), we have

$$
\sigma \beta_{1}=\tau \alpha_{1} .
$$

Then (4.15) is implied by (4.18) and (4.19). Note that the equations of the coefficients $\xi_{r} \psi_{s}, \zeta_{r} \psi_{s}, \xi_{r} \phi_{s}$ and $\zeta_{r} \phi_{s}$ in $\partial_{\mathcal{Z}}\left(R_{2}\right)=\partial_{\mathcal{Y}}\left(R_{3}\right)$ are implied by (4.16), (4.18) and (4.19).

According to (4.14) and the second equation in (4.17),

$$
f_{\mathcal{Y}}+h_{\mathcal{Z}}=\gamma_{1}
$$

a function of $t$. Under the conditions in (4.16), the first equation in (4.17), and (4.18)(4.20), $\partial_{\mathcal{Y}}\left(R_{1}\right)=\partial_{\mathcal{X}}\left(R_{2}\right)$ becomes

$$
\alpha^{\prime} h_{\mathcal{Z}}-\beta^{\prime} h_{\mathcal{Y}} \sin \alpha=\alpha^{\prime \prime}
$$

$\partial_{\mathcal{Z}}\left(R_{1}\right)=\partial_{\mathcal{X}}\left(R_{3}\right)$ is equivalent to

$$
\beta^{\prime} h_{\mathcal{Z}} \sin \alpha+\alpha^{\prime} h_{y}=\beta^{\prime} \gamma_{1} \sin \alpha-\left(\beta^{\prime} \sin \alpha\right)^{\prime}-2 \alpha^{\prime} \beta^{\prime} \cos \alpha
$$


and $\partial_{\mathcal{Z}}\left(R_{2}\right)=\partial_{\mathcal{Y}}\left(R_{3}\right)$ says

$$
\left(f f_{\mathcal{Y}}+h f_{\mathcal{Z}}\right)_{\mathcal{Z}}=\left(f h_{\mathcal{Y}}+h h_{\mathcal{Z}}\right)_{\mathcal{Y}}+2 \beta^{\prime} \gamma_{1} \cos \alpha .
$$

By (4.18) and (4.20)-(4.22), we assume $f_{\mathcal{Y}}, f_{\mathcal{Z}}, h_{\mathcal{Y}}$ and $h_{\mathcal{Z}}$ are functions of $t$. Then (4.23) can be written as

$$
\left(f_{\mathcal{Y}}+h_{\mathcal{Z}}\right) f_{\mathcal{Z}}=\left(f_{\mathcal{Y}}+h_{\mathcal{Z}}\right) h_{\mathcal{Y}}+2 \beta^{\prime} \gamma_{1} \cos \alpha
$$

which is implied by (4.18) and (4.20). Solving (4.21) and (4.22), we get

$$
\begin{gathered}
h_{\mathcal{Y}}=\frac{\alpha^{\prime} \beta^{\prime} \gamma_{1} \sin \alpha-\left(\alpha^{\prime} \beta^{\prime} \sin \alpha\right)^{\prime}-2\left(\alpha^{\prime}\right)^{2} \beta^{\prime} \cos \alpha}{\left(\alpha^{\prime}\right)^{2}+\left(\beta^{\prime}\right)^{2} \sin ^{2} \alpha} \\
h_{\mathcal{Z}}=\frac{\alpha^{\prime} \alpha^{\prime \prime}+\left(\beta^{\prime}\right)^{2} \gamma_{1} \sin ^{2} \alpha-\left(\beta^{\prime} \sin \alpha\right)\left(\beta^{\prime} \sin \alpha\right)^{\prime}-\alpha^{\prime}\left(\beta^{\prime}\right)^{2} \sin 2 \alpha}{\left(\alpha^{\prime}\right)^{2}+\left(\beta^{\prime}\right)^{2} \sin ^{2} \alpha} .
\end{gathered}
$$

Moreover,

$$
f_{\mathcal{Y}}=\frac{\gamma_{1}\left(\alpha^{\prime}\right)^{2}-\alpha^{\prime} \alpha^{\prime \prime}+\left(\beta^{\prime} \sin \alpha\right)\left(\beta^{\prime} \sin \alpha\right)^{\prime}+\alpha^{\prime}\left(\beta^{\prime}\right)^{2} \sin 2 \alpha}{\left(\alpha^{\prime}\right)^{2}+\left(\beta^{\prime}\right)^{2} \sin ^{2} \alpha}
$$

by (4.20) and (4.26), and

$$
f_{\mathcal{Z}}=\frac{\alpha^{\prime} \beta^{\prime} \gamma_{1} \sin \alpha-\left(\alpha^{\prime} \beta^{\prime} \sin \alpha\right)^{\prime}+2\left(\beta^{\prime}\right)^{2} \sin ^{2} \alpha \cos \alpha}{\left(\alpha^{\prime}\right)^{2}+\left(\beta^{\prime}\right)^{2} \sin ^{2} \alpha}
$$

by (4.18) and (4.25). With the above data, we take

$$
f=f_{\mathcal{Y}} \mathcal{Y}+f_{\mathcal{Z}} \mathcal{Z}, \quad h=h_{\mathcal{Y}} \mathcal{Y}+h_{\mathcal{Z}} \mathcal{Z}
$$

by the transformations of the type in (1.8) and (1.9). Furthermore, (4.17), (4.19) and the first equation in (4.17) yield $r+s+1 \in 2 \mathbb{Z}$,

$$
\begin{gathered}
\alpha_{1}=\varphi \alpha^{\prime}, \quad \gamma= \pm \varphi \sqrt{\left(\alpha^{\prime}\right)^{2}+\left(\beta^{\prime}\right)^{2} \sin ^{2} \alpha}, \\
\beta_{1}=\varphi \beta^{\prime} \sin \alpha, \quad \sigma=\mu \alpha^{\prime}, \quad \tau=\mu \beta^{\prime} \sin \alpha .
\end{gathered}
$$

In particular, $\alpha, \beta, \gamma_{1}, \varphi$ and $\mu$ are arbitrary functions of $t$. According (4.9)-(4.11), the pressure

$$
\begin{aligned}
p & =\rho\left\{\gamma \mu \varphi^{-1}\left[\left(\gamma^{\prime}-\gamma \gamma_{1}\right) \mathcal{X} \zeta_{r} \phi_{s}-\left(\left(\varphi \alpha^{\prime}\right)^{\prime} \mathcal{Y}+\left(\varphi \beta^{\prime} \sin \alpha\right)^{\prime} \mathcal{Z}+\varphi\left(\alpha^{\prime} f+\beta^{\prime} h \sin \alpha\right)\right) \xi_{r} \psi_{s}\right]\right. \\
& +(-1)^{s} \varphi^{-1}\left[(\gamma \mu)^{\prime}-\gamma \mu \varphi^{\prime} \varphi^{-1}\right] \zeta_{r} \psi_{s}+2 \mu\left(\left(\alpha^{\prime}\right)^{2}+\left(\beta^{\prime}\right)^{2} \sin ^{2} \alpha\right) \xi_{r} \phi_{s}+2 \alpha^{\prime} f \mathcal{X} \\
& +\frac{\left(\alpha^{\prime}\right)^{2}+\left(\beta^{\prime}\right)^{2} \sin ^{2} \alpha+\gamma_{1}^{\prime}-\gamma_{1}^{2}}{2} \mathcal{X}^{2}+2 \beta^{\prime} h \mathcal{X} \sin \alpha+\left[\left(\beta^{\prime} \sin \alpha\right)^{\prime}-\alpha^{\prime} \beta^{\prime} \cos \alpha\right] \mathcal{X} \mathcal{Z} \\
& +\left(\frac{\left(\beta^{\prime}\right)^{2}}{2} \sin 2 \alpha+\alpha^{\prime \prime}\right) \mathcal{X Y}-\frac{1}{2} \gamma^{4} \mu^{2} \varphi^{-2}\left[\left(\delta_{r, 1}+4 a \delta_{r, 0}\right) \phi_{s}^{2}+\left(4 b \delta_{s, 0}+\delta_{s, 1}\right) \xi_{r}^{2}\right] \\
& +\left[\left(\beta^{\prime} \cos \alpha\right)^{\prime}+\alpha^{\prime} \beta^{\prime} \sin \alpha-f_{\mathcal{Z} t}-f_{\mathcal{Y}} f_{\mathcal{Z}}-h_{\mathcal{Y}} h_{\mathcal{Z}}\right] \mathcal{Y Z}+\frac{\left(\beta^{\prime}\right)^{2}-h_{\mathcal{Z} t}-f_{\mathcal{Z}}^{2}-h_{\mathcal{Z}}^{2}}{2} \mathcal{Z}^{2} \\
& \left.+\frac{\left(\alpha^{\prime}\right)^{2}+\left(\beta^{\prime}\right)^{2} \cos \alpha-f_{\mathcal{Y} t}-f_{\mathcal{Y}}^{2}-h_{\mathcal{Y}}^{2}}{2} \mathcal{Y}^{2}\right\}
\end{aligned}
$$

modulo the transformation in (1.10). By (3.3) and (3.5), we have the following theorem: 
Theorem 4.1. Let $\alpha, \beta, \gamma_{1}, \varphi$ and $\mu$ be arbitrary functions of $t$ such that $\varphi \neq 0$ and $\left(\alpha^{\prime}\right)^{2}+\left(\beta^{\prime}\right)^{2} \sin ^{2} \alpha \neq 0$. Take any real constants $a$ and $b$. The notation $\mathcal{X}, \mathcal{Y}$ and $\mathcal{Z}$ are defined in (3.5) via (3.1), and the notation $\xi_{r}, \zeta_{r}, \phi_{r}$ and $\psi_{r}$ are defined in (4.4)-(4.7) with $\alpha_{1}, \beta_{1}$ and $\gamma$ given in (4.30) and (4.31). Moreover, $f_{\mathcal{Y}}, f_{\mathcal{Z}}, h_{\mathcal{Y}}, h_{\mathcal{Z}}$ and $f, h$ are given in (4.25)-(4.29). Assume $(r, s) \in\{(0,1),(1,0)\}$. We have the following solution of the Navier-Stokes equations (1.1)-(1.4):

$$
\begin{gathered}
u=-\alpha^{\prime}(\mathcal{X} \sin \alpha+\mathcal{Y} \cos \alpha)-\left(f+\mu \alpha^{\prime} \gamma \xi_{r} \psi_{s}\right) \sin \alpha \\
-\left(\gamma_{1} \mathcal{X}+\varphi \mu\left(\left(\alpha^{\prime}\right)^{2}+\left(\beta^{\prime}\right)^{2} \sin ^{2} \alpha\right) \zeta_{r} \phi_{s}\right) \cos \alpha, \\
v=\left(f \cos \alpha-\beta^{\prime} \mathcal{Z}\right) \cos \beta-\left(\alpha^{\prime} \sin \alpha \cos \beta+\beta^{\prime} \cos \alpha \sin \beta\right) \mathcal{Y} \\
-\left(\gamma_{1} \mathcal{X}+\varphi \mu\left(\left(\alpha^{\prime}\right)^{2}+\left(\beta^{\prime}\right)^{2} \sin ^{2} \alpha\right) \zeta_{r} \phi_{s}\right) \sin \alpha \cos \beta-h \sin \beta \\
+\left(\alpha^{\prime} \cos \alpha \cos \beta-\beta^{\prime} \sin \alpha \sin \beta\right)\left(\mathcal{X}+\gamma \mu \xi_{r} \psi_{s}\right), \\
w=\left(\beta^{\prime} \cos \alpha \cos \beta-\alpha^{\prime} \sin \alpha \sin \beta\right) \mathcal{Y}+\left(f \cos \alpha-\beta^{\prime} \mathcal{Z}\right) \sin \beta \\
-\left(\gamma_{1} \mathcal{X}+\varphi \mu\left(\left(\alpha^{\prime}\right)^{2}+\left(\beta^{\prime}\right)^{2} \sin ^{2} \alpha\right) \zeta_{r} \phi_{s}\right) \sin \alpha \sin \beta+h \cos \beta \\
+\left(\alpha^{\prime} \cos \alpha \sin \beta+\beta^{\prime} \sin \alpha \cos \beta\right)\left(\mathcal{X}+\gamma \mu \xi_{r} \psi_{s}\right),
\end{gathered}
$$

and $p$ is given in (4.32). The above solution is globally analytic in $x, y, z$.

Let $\gamma_{1}, \gamma_{2}$ be functions of $t$ and let $a, b, c$ be real numbers. Denote

$$
\begin{array}{cc}
\phi_{0}=e^{\gamma_{1} \mathcal{Y}+\gamma_{2} \mathcal{Z}}-a e^{-\gamma_{1} \mathcal{Y}-\gamma_{2} \mathcal{Z}}, & \phi_{1}=\sin \left(\gamma_{1} \mathcal{Y}+\gamma_{2} \mathcal{Z}\right), \\
\psi_{0}=e^{\gamma_{1} \mathcal{Y}+\gamma_{2} \mathcal{Z}}+a e^{-\gamma_{1} \mathcal{Y}-\gamma_{2} \mathcal{Z}}, & \psi_{1}=\cos \left(\gamma_{1} \mathcal{Y}+\gamma_{2} \mathcal{Z}\right), \\
\xi_{0}=b e^{\gamma_{1} \mathcal{Y}+\gamma_{2} \mathcal{Z}}-c e^{-\gamma_{1} \mathcal{Y}-\gamma_{2} \mathcal{Z}}, & \xi_{1}=c \sin \left(\gamma_{1} \mathcal{Y}+\gamma_{2} \mathcal{Z}+b\right), \\
\zeta_{0}=b e^{\gamma_{1} \mathcal{Y}+\gamma_{2} \mathcal{Z}}+c e^{-\gamma_{1} \mathcal{Y}-\gamma_{2} \mathcal{Z}}, & \zeta_{1}=c \cos \left(\gamma_{1} \mathcal{Y}+\gamma_{2} \mathcal{Z}+b\right) .
\end{array}
$$

Suppose that $\sigma, \tau$ are functions of $t$ and $f, k, h$ are functions in $t, \mathcal{X}, \mathcal{Y}, \mathcal{Z}$ such that $h$ and $g$ are linear in $\mathcal{X}, Y, \mathcal{Z}$ and

$$
f_{\mathcal{X}}+k_{\mathcal{Y}}+h_{\mathcal{Z}}=0
$$

Motivated from the above solution, we consider the solution of the form

$$
\begin{gathered}
\mathcal{U}=-\alpha^{\prime} \mathcal{Y}-\beta^{\prime} \mathcal{Z} \sin \alpha+f-\left(\gamma_{1}^{2}+\gamma_{2}^{2}\right)\left(\tau \zeta_{r} \mathcal{X}+\sigma \psi_{r} \mathcal{X}^{2}\right) \\
\mathcal{V}=\alpha^{\prime} \mathcal{X}-\beta^{\prime} \mathcal{Z} \cos \alpha+k+\gamma_{1}\left(\tau \xi_{r}+2 \sigma \phi_{r} \mathcal{X}\right) \\
\mathcal{W}=\beta^{\prime}(\mathcal{X} \sin \alpha+\mathcal{Y} \cos \alpha)+h+\gamma_{2}\left(\tau \xi_{r}+2 \sigma \phi_{r} \mathcal{X}\right)
\end{gathered}
$$

where $\gamma_{1}$ and $\gamma_{2}$ are functions of $t$.

For convenience of computation, we denote

$$
\gamma=\gamma_{1}^{2}+\gamma_{2}^{2}, \quad f^{*}=f-f_{x} \mathcal{X} \quad \Delta_{1}=\partial_{\mathcal{Y}}^{2}+\partial_{\mathcal{Z}}^{2} .
$$


Now (4.1) becomes

$$
\begin{aligned}
R_{1} & =-\alpha^{\prime \prime} \mathcal{Y}-\left(\beta^{\prime} \sin \alpha\right)^{\prime} \mathcal{Z}+f_{t}-(-1)^{r} \gamma\left(\gamma_{1}^{\prime} \mathcal{Y}+\gamma_{2}^{\prime} \mathcal{Z}\right)\left(\tau \xi_{r} \mathcal{X}+\sigma \phi_{r} \mathcal{X}^{2}\right) \\
& +\left((-1)^{r} \nu \gamma^{2} \tau-(\gamma \tau)^{\prime}\right) \zeta_{r} \mathcal{X}+\left(f-\gamma\left(\tau \zeta_{r} \mathcal{X}+\sigma \psi_{r} \mathcal{X}^{2}\right)\right)\left(f \mathcal{X}-\gamma\left(\tau \zeta_{r}+2 \sigma \psi_{r} \mathcal{X}\right)\right) \\
& +\left(k+\gamma_{1}\left(\tau \xi_{r}+2 \sigma \phi_{r} \mathcal{X}\right)\right)\left[f_{\mathcal{Y}}-2 \alpha^{\prime}-(-1)^{r} \gamma \gamma_{1}\left(\tau \xi_{r} \mathcal{X}+\sigma \phi_{r} \mathcal{X}^{2}\right)\right]-\nu \Delta_{1}(f) \\
& +\left(h+\gamma_{2}\left(\tau \xi_{r}+2 \sigma \phi_{r} \mathcal{X}\right)\right)\left[f_{\mathcal{Z}}-2 \beta^{\prime} \sin \alpha-(-1)^{r} \gamma \gamma_{2}\left(\tau \xi_{r} \mathcal{X}+\sigma \phi_{r} \mathcal{X}^{2}\right)\right]+2 \nu \gamma \sigma \psi_{r} \\
& -\alpha^{\prime}\left(\alpha^{\prime} \mathcal{X}-\beta^{\prime} \mathcal{Z} \cos \alpha\right)-\left(\beta^{\prime}\right)^{2}(\mathcal{X} \sin \alpha+\mathcal{Y} \cos \alpha) \sin \alpha+\left((-1)^{r} \nu \gamma^{2} \sigma-(\gamma \sigma)^{\prime}\right) \psi_{r} \mathcal{X}^{2} \\
& =-\left(\left(\alpha^{\prime}\right)^{2}+\left(\beta^{\prime}\right)^{2} \sin ^{2} \alpha\right) \mathcal{X}-\left(\alpha^{\prime \prime}+2^{-1}\left(\beta^{\prime}\right)^{2} \sin 2 \alpha\right) \mathcal{Y}+\left(\alpha^{\prime} \beta^{\prime} \cos \alpha-\left(\beta^{\prime} \sin \alpha\right)^{\prime}\right) \mathcal{Z} \\
& +\gamma^{2}\left[\tau^{2}\left(4 b \delta_{0, r}+c \delta_{1, r}\right) c \mathcal{X}+3 \sigma \tau\left(2 \delta_{0, r}(a b+c)+\delta_{1, r} c \cos b\right) \mathcal{X}^{2}+2 \sigma^{2}\left(4 a \delta_{0, r}+\delta_{1, r}\right) \mathcal{X}^{3}\right] \\
& -(-1)^{r} \gamma\left(\gamma_{1}^{\prime} \mathcal{Y}+\gamma_{2}^{\prime} \mathcal{Z}+k \gamma_{1}+h \gamma_{2}\right)\left(\tau \xi_{r} \mathcal{X}+\sigma \phi_{r} \mathcal{X}^{2}\right)+f f_{\mathcal{X}}+k f_{\mathcal{Y}}+h f_{\mathcal{Z}} \\
& +\left((-1)^{r} \nu \gamma^{2} \sigma-(\gamma \sigma)^{\prime}-3 \gamma \sigma f_{\mathcal{X}}\right) \psi_{r} \mathcal{X}^{2}+\nu\left(2 \sigma \psi_{r}-\Delta_{1}(f)\right)-\gamma \tau f^{*} \zeta_{r} \\
& -\left[\left((\gamma \tau)^{\prime}+2 \gamma \tau f_{\mathcal{X}}-(-1)^{r} \nu \gamma^{2} \tau\right) \zeta_{r}+2 \gamma \sigma f^{*} \psi_{r}\right] \mathcal{X}+f_{t} \\
& +\left(\gamma_{1}\left(f_{\mathcal{Y}}-2 \alpha^{\prime}\right)+\gamma_{2}\left(f_{\mathcal{Z}}-2 \beta^{\prime} \sin \alpha\right)\right)\left(\tau \xi_{r}+2 \sigma \phi_{r} \mathcal{X}\right) .
\end{aligned}
$$

To solve (3.14), we assume

$$
\gamma_{1}^{\prime} \mathcal{Y}+\gamma_{2}^{\prime} \mathcal{Z}+k \gamma_{1}+h \gamma_{2}=0
$$

and

$$
(-1)^{r} \nu \gamma^{2} \sigma-(\gamma \sigma)^{\prime}-3 \gamma \sigma f_{\mathcal{X}}=0
$$

Moreover, (4.2) and (4.3) become

$$
\begin{aligned}
R_{2} & =\alpha^{\prime \prime} \mathcal{X}-\left(\beta^{\prime} \cos \alpha\right)^{\prime} \mathcal{Z}+\left(\left(\gamma_{1} \tau\right)^{\prime}-(-1)^{r} \nu \gamma \gamma_{1} \tau\right) \xi_{r}+2\left(\left(\gamma_{1} \sigma\right)^{\prime}-(-1)^{r} \nu \gamma \gamma_{1} \sigma\right) \phi_{r} \mathcal{X} \\
& +k_{t}+\left(\gamma_{1}^{\prime} \mathcal{Y}+\gamma_{2}^{\prime} \mathcal{Z}\right) \gamma_{1}\left(\tau \zeta_{r}+2 \sigma \psi_{r} \mathcal{X}\right)+\left(f-\gamma\left(\tau \zeta_{r} \mathcal{X}+\sigma \psi_{r} \mathcal{X}^{2}\right)\right)\left(2 \alpha^{\prime}+k_{\mathcal{X}}+2 \gamma_{1} \sigma \phi_{r}\right) \\
& +\left(k+\gamma_{1}\left(\tau \xi_{r}+2 \sigma \phi_{r} \mathcal{X}\right)\right)\left(k_{\mathcal{Y}}+\gamma_{1}^{2}\left(\tau \zeta_{r}+2 \sigma \psi_{r} \mathcal{X}\right)\right)-\left(\beta^{\prime}\right)^{2}(\mathcal{X} \sin \alpha+\mathcal{Y} \cos \alpha) \cos \alpha \\
& -\alpha^{\prime}\left(\alpha^{\prime} \mathcal{Y}+\beta^{\prime} \mathcal{Z} \sin \alpha\right)+\left(h+\gamma_{2}\left(\tau \xi_{r}+2 \sigma \phi_{r} \mathcal{X}\right)\right)\left(k_{\mathcal{Z}}-2 \beta^{\prime} \cos \alpha+\gamma_{1} \gamma_{2}\left(\tau \zeta_{r}+2 \sigma \psi_{r} \mathcal{X}\right)\right) \\
& =\left(\alpha^{\prime \prime}-2^{-1}\left(\beta^{\prime}\right)^{2} \sin 2 \alpha+f_{\mathcal{X}}\left(2 \alpha^{\prime}+k_{\mathcal{X}}\right)\right) \mathcal{X}-\left(\left(\alpha^{\prime}\right)^{2}+\left(\beta^{\prime}\right)^{2} \cos ^{2} \alpha\right) \mathcal{Y}+k_{t}+k k_{\mathcal{Y}} \\
& +\left[\tau\left(\gamma_{1} k_{\mathcal{Y}}+\gamma_{2}\left(k_{\mathcal{Z}}-2 \beta^{\prime} \cos \alpha\right)\right)+\left(\gamma_{1} \tau\right)^{\prime}-(-1)^{r} \nu \gamma \gamma_{1} \tau\right] \xi_{r}-\left(\left(\beta^{\prime} \cos \alpha\right)^{\prime}+\alpha^{\prime} \beta^{\prime} \sin \alpha\right) \mathcal{Z} \\
& +\gamma \sigma\left(2 \gamma_{1} \sigma \phi_{r}-2 \alpha^{\prime}-k_{\mathcal{X}}\right) \psi_{r} \mathcal{X}^{2}+f^{*}\left(2 \alpha^{\prime}+k_{\mathcal{X}}+2 \gamma_{1} \sigma \phi_{r}\right)+h\left(k_{\mathcal{Z}}-2 \beta^{\prime} \cos \alpha\right) \\
& +\gamma \gamma_{1} \tau^{2} \xi_{r} \zeta_{r}+\left\{2 \gamma \gamma_{1} \sigma \tau \xi_{r} \psi_{r}+2\left[\left(\gamma_{1} \sigma\right)^{\prime}-\sigma\left(\gamma_{1}\left(h_{\mathcal{Z}}+(-1)^{r} \nu \gamma\right)\right.\right.\right. \\
& \left.\left.\left.+\gamma_{2}\left(2 \beta^{\prime} \cos \alpha-k_{\mathcal{Z}}\right)\right)\right] \phi_{r}-\gamma \tau\left(2 \alpha^{\prime}+k_{\mathcal{X}}\right) \zeta_{r}\right\} \mathcal{X}
\end{aligned}
$$




$$
\begin{aligned}
R_{3} & =\left(\beta^{\prime} \sin \alpha\right)^{\prime} \mathcal{X}+\left(\beta^{\prime} \cos \alpha\right)^{\prime} \mathcal{Y}+\left(\gamma_{2} \tau\right)^{\prime} \xi_{r}+2\left(\gamma_{2} \sigma\right)^{\prime} \phi_{r} \mathcal{X}-(-1)^{r} \nu \gamma_{2}^{3}\left(\tau \xi_{r}+\sigma \phi_{r} \mathcal{X}\right) \\
& +\left(\gamma_{1}^{\prime} \mathcal{Y}+\gamma_{2}^{\prime} \mathcal{Z}\right) \gamma_{2}\left(\tau \zeta_{r}+2 \sigma \psi_{r} \mathcal{X}\right)+\left(f-\gamma\left(\tau \zeta_{r} \mathcal{X}+\sigma \psi_{r} \mathcal{X}^{2}\right)\right)\left(2 \beta^{\prime} \sin \alpha+h_{\mathcal{X}}+2 \gamma_{2} \sigma \phi_{r}\right) \\
& +\left(k+\gamma_{1}\left(\tau \xi_{r}+2 \sigma \phi_{r} \mathcal{X}\right)\right)\left(2 \beta^{\prime} \cos \alpha+h_{\mathcal{Y}}+\gamma_{1} \gamma_{2}\left(\tau \zeta_{r}+2 \sigma \psi_{r} \mathcal{X}\right)\right)-\left(\beta^{\prime}\right)^{2} \mathcal{Z}+h_{t} \\
& +\alpha^{\prime} \beta^{\prime}(\mathcal{X} \cos \alpha-\mathcal{Y} \sin \alpha)+\left(h+\gamma_{2}\left(\tau \xi_{r}+2 \sigma \phi_{r} \mathcal{X}\right)\right)\left(h_{\mathcal{Z}}+\gamma_{2}^{2}\left(\tau \zeta_{r}+2 \sigma \psi_{r} \mathcal{X}\right)\right) \\
& =\left[\left(\beta^{\prime} \sin \alpha\right)^{\prime}+\alpha^{\prime} \beta^{\prime} \cos \alpha+f_{\mathcal{X}}\left(2 \beta^{\prime} \sin \alpha+h_{\mathcal{X}}\right)\right] \mathcal{X}+\left[\left(\beta^{\prime} \cos \alpha\right)^{\prime}-\alpha^{\prime} \beta^{\prime} \sin \alpha\right] \mathcal{Y} \\
& +\left[\left(\gamma_{2} \tau\right)^{\prime}+\left(\gamma_{1}\left(2 \beta^{\prime} \cos \alpha+h_{\mathcal{Y}}\right)+\gamma_{2} h_{\mathcal{Z}}-(-1)^{r} \nu \gamma \gamma_{2}\right) \tau\right] \xi_{r}+\left\{2 \gamma \gamma_{2} \tau \sigma \xi_{r} \psi_{r}+2\left[\left(\gamma_{2} \sigma\right)^{\prime}\right.\right. \\
& \left.\left.-\gamma_{2} \sigma\left(k \mathcal{Y}+(-1)^{r} \nu \gamma\right)+\gamma_{1} \sigma\left(2 \beta^{\prime} \cos \alpha+h_{\mathcal{Y}}\right)\right] \phi_{r}-\gamma \tau\left(2 \beta^{\prime} \sin \alpha+h_{\mathcal{X}}\right) \zeta_{r}\right\} \mathcal{X} \\
& +f^{*}\left(2 \beta^{\prime} \sin \alpha+h_{\mathcal{X}}+2 \gamma_{2} \sigma \phi_{r}\right)+k\left(2 \beta^{\prime} \cos \alpha+h_{\mathcal{Y}}\right)+h_{t}+h h_{\mathcal{Z}}+\gamma \gamma_{2} \tau^{2} \xi_{r} \zeta_{r} \\
& +\gamma \sigma \psi_{r}\left(2 \gamma_{2} \sigma \phi_{r}-2 \beta^{\prime} \sin \alpha-h_{\mathcal{X}}\right) \mathcal{X}^{2}-\left(\beta^{\prime}\right)^{2} \mathcal{Z}
\end{aligned}
$$

By the coefficients of $\mathcal{X}^{2}$ in $\partial_{\mathcal{Z}}\left(R_{2}\right)=\partial_{\mathcal{Y}}\left(R_{3}\right)$, we have

$$
\gamma_{2}\left(2 \alpha^{\prime}+k_{\mathcal{X}}\right)=\gamma_{1}\left(2 \beta^{\prime} \sin \alpha+h_{\mathcal{X}}\right) .
$$

According to (4.46),

$$
k_{\mathcal{X}} \gamma_{1}+h_{\mathcal{X}} \gamma_{2}=0, \quad \gamma_{1}^{\prime}+\gamma_{1} k_{\mathcal{Y}}+\gamma_{2} h_{\mathcal{Y}}=0, \quad \gamma_{2}^{\prime}+\gamma_{1} k_{\mathcal{Z}}+\gamma_{2} h_{\mathcal{Z}}=0 .
$$

Solving (4.50) and the first equation in (4.51), we obtain

$$
k_{\mathcal{X}}=2 \gamma^{-1} \gamma_{2}\left(\beta^{\prime} \gamma_{1} \sin \alpha-\alpha^{\prime} \gamma_{2}\right), \quad h_{\mathcal{X}}=-2 \gamma^{-1} \gamma_{1}\left(\beta^{\prime} \gamma_{1} \sin \alpha-\alpha^{\prime} \gamma_{2}\right)
$$

Moreover, the coefficients of $\mathcal{X}$ in $\partial_{\mathcal{Z}}\left(R_{2}\right)=\partial_{\mathcal{Y}}\left(R_{3}\right)$ give

$$
\gamma_{1}^{\prime} \gamma_{2}-\gamma_{1} \gamma_{2}^{\prime}+\gamma_{1} \gamma_{2}\left(k_{\mathcal{Y}}-h_{\mathcal{Z}}\right)+\gamma_{2}^{2} k_{\mathcal{Z}}-\gamma_{1}^{2} h_{\mathcal{Y}}-2 \gamma \beta^{\prime} \cos \alpha=0 .
$$

By (4.51), the above equation can be rewritten as

$$
k_{\mathcal{Z}}-h_{\mathcal{Y}}=2 \beta^{\prime} \cos \alpha
$$

Furthermore, (4.50) and the coefficients of $\mathcal{X}^{0}$ in $\partial_{\mathcal{Z}}\left(R_{2}\right)=\partial_{\mathcal{Y}}\left(R_{3}\right)$ show that $f$ is a function of $t$ and $\gamma_{1} \mathcal{Y}+\gamma_{2} \mathcal{Z}$. According to the coefficients of $\mathcal{X}$ in $\partial_{\mathcal{Y}}\left(R_{1}\right)=\partial_{\mathcal{X}}\left(R_{2}\right)$ and $\partial_{\mathcal{Z}}\left(R_{1}\right)=\partial_{\mathcal{X}}\left(R_{3}\right)$, we take

$$
f^{*}=\varphi \vartheta_{r}+\sigma \tilde{\varpi} \phi_{r}+\alpha_{1}
$$

where $\varphi$ and $\alpha_{1}$ are functions of $t$, and

$$
\tilde{\varpi}=\gamma_{1} \mathcal{Y}+\gamma_{2} \mathcal{Z}, \quad \vartheta_{0}=b_{1} e^{\tilde{\varpi}}-c_{1} e^{-\tilde{\varpi}}, \quad \vartheta_{1}=c_{1} \sin \left(\tilde{\varpi}+b_{1}\right)
$$

for $b_{1}, c_{1} \in \mathbb{R}$.

Now the coefficients of $\mathcal{X}$ in $\partial_{\mathcal{Y}}\left(R_{1}\right)=\partial_{\mathcal{X}}\left(R_{2}\right)$ and $\partial_{\mathcal{X}}\left(R_{1}\right)=\partial_{\mathcal{X}}\left(R_{3}\right)$ give

$$
\mathcal{T}\left[2 \alpha_{1} \gamma \sigma \psi_{r}+\left((\gamma \tau)^{\prime}+2 \gamma \tau f_{\mathcal{X}}-(-1)^{r} \nu \gamma^{2} \tau\right) \zeta_{r}\right]=0, \quad \mathcal{T}=\partial_{\mathcal{Y}}, \partial_{\mathcal{Z}} .
$$


Moreover, the coefficients of $\mathcal{X}^{0}$ in $\partial_{\mathcal{Y}}\left(R_{1}\right)=\partial_{\mathcal{X}}\left(R_{2}\right)$ and $\partial_{\mathcal{X}}\left(R_{1}\right)=\partial_{\mathcal{X}}\left(R_{3}\right)$ yield

$$
\begin{gathered}
{\left[\left(\left(f_{\mathcal{X}}-(-1)^{r} \gamma \nu\right) \varphi+\varphi^{\prime}\right) \vartheta_{r}+\left(\left(f_{\mathcal{X}}-(-1)^{r} \nu \gamma\right) \sigma+\sigma^{\prime}\right) \tilde{\varpi} \phi_{r}-\alpha_{1} \gamma \tau \zeta_{r}\right] \mathcal{Y}} \\
=2\left[\left(\gamma_{1} \sigma\right)^{\prime}-\sigma\left(\gamma_{1}\left(h_{\mathcal{Z}}+(-1)^{r} \nu \gamma\right)-\gamma_{2} h_{\mathcal{Y}}\right)\right] \phi_{r} \\
+2 \alpha^{\prime \prime}+2 \alpha^{\prime} f_{\mathcal{X}}+k_{\mathcal{X} t}+h_{\mathcal{X}} h_{\mathcal{Y}}-k_{\mathcal{X}} h_{\mathcal{Z}}, \\
{\left[\left(\left(f_{\mathcal{X}}-(-1)^{r} \gamma \nu\right) \varphi+\varphi^{\prime}\right) \vartheta_{r}+\left(\left(f_{\mathcal{X}}-(-1)^{r} \nu \gamma\right) \sigma+\sigma^{\prime}\right) \tilde{\varpi} \phi_{r}-\alpha_{1} \gamma \tau \zeta_{r}\right] \mathcal{Z}} \\
=2\left(\beta^{\prime} \sin \alpha\right)^{\prime}+2 \beta^{\prime} f_{\mathcal{X}} \sin \alpha+h_{\mathcal{X} t}-k_{\mathcal{Y}} h_{\mathcal{X}}+k_{\mathcal{X}} k_{\mathcal{Z}} \\
+2\left[\left(\gamma_{2} \sigma\right)^{\prime}-\gamma_{2} \sigma\left(k_{\mathcal{Y}}+(-1)^{r} \nu \gamma\right)+\gamma_{1} \sigma k_{\mathcal{Z}}\right] \phi_{r} .
\end{gathered}
$$

Thus we have

$$
\begin{gathered}
2 \alpha^{\prime \prime}+2 \alpha^{\prime} f_{\mathcal{X}}+k_{\mathcal{X} t}+h_{\mathcal{X}} h_{\mathcal{Y}}-k_{\mathcal{X}} h_{\mathcal{Z}}=0, \\
2\left(\beta^{\prime} \sin \alpha\right)^{\prime}+2 \beta^{\prime} f_{\mathcal{X}} \sin \alpha+h_{\mathcal{X} t}-k_{\mathcal{Y}} h_{\mathcal{X}}+k_{\mathcal{X}} k_{\mathcal{Z}}=0 .
\end{gathered}
$$

For simplicity, we only consider two special cases as follows.

Case 1. $\vartheta_{r}=\zeta_{r}, \sigma=0, \gamma_{1}=\alpha^{\prime} \mu$ and $\gamma_{2}=\beta^{\prime} \mu \sin \alpha$.

In this case,

$$
k_{\mathcal{X}}=h_{\mathcal{X}}=0
$$

by (4.52). According to (4.60) and (4.61), we have

$$
\beta^{\prime} \sin \alpha=d \alpha^{\prime}, \quad f_{\mathcal{X}}=-\frac{\alpha^{\prime \prime}}{\alpha^{\prime}} .
$$

Moreover, (4.51) becomes

$$
k_{\mathcal{Y}}+d h_{\mathcal{Y}}=-\frac{\left(\mu \alpha^{\prime}\right)^{\prime}}{\mu \alpha^{\prime}}, \quad k_{\mathcal{Z}}+d h_{\mathcal{Z}}=-d \frac{\left(\mu \alpha^{\prime}\right)^{\prime}}{\mu \alpha^{\prime}} .
$$

According to (4.40) and (4.54),

$$
h_{\mathcal{Z}}=\frac{\alpha^{\prime \prime}}{\alpha^{\prime}}-k_{\mathcal{Y}}, \quad h_{\mathcal{Y}}=k_{\mathcal{Z}}-2 \beta^{\prime} \cos \alpha
$$

Substituting (4.65) into (4.64), we obtain

$$
k_{\mathcal{Y}}+d k_{\mathcal{Z}}=2 d \beta^{\prime} \cos \alpha-\frac{\left(\mu \alpha^{\prime}\right)^{\prime}}{\mu \alpha^{\prime}}, \quad k_{\mathcal{Z}}-d k_{\mathcal{Y}}=-d \frac{\left(\mu \alpha^{\prime}\right)^{\prime}+\alpha^{\prime \prime} \mu}{\mu \alpha^{\prime}} .
$$

For convenience of computation, we write

$$
\mu=\frac{\sqrt{\beta_{1}^{\prime}}}{\alpha^{\prime}} \Longrightarrow \gamma=\left(1+d^{2}\right) \beta_{1}^{\prime}, \quad \gamma_{1}=\sqrt{\beta_{1}}, \quad \gamma_{2}=d \sqrt{\beta_{1}} .
$$

From (4.66),

$$
\begin{gathered}
k_{\mathcal{Y}}=\frac{1}{1+d^{2}}\left(2 d^{2} \alpha^{\prime} \cot \alpha+\left(d^{2}-1\right) \frac{\beta_{1}^{\prime \prime}}{2 \beta_{1}^{\prime}}+d^{2} \frac{\alpha^{\prime \prime}}{\alpha^{\prime}}\right), \\
k_{\mathcal{Z}}=\frac{d}{1+d^{2}}\left(2 d^{2} \alpha^{\prime} \cot \alpha-\frac{\beta_{1}^{\prime \prime}}{\beta_{1}^{\prime}}-\frac{\alpha^{\prime \prime}}{\alpha^{\prime}}\right) .
\end{gathered}
$$


By (4.65),

$$
\begin{gathered}
h_{\mathcal{Z}}=\frac{1}{1+d^{2}}\left(\left(1-d^{2}\right) \frac{\beta_{1}^{\prime \prime}}{2 \beta_{1}^{\prime}}+\frac{\alpha^{\prime \prime}}{\alpha^{\prime}}-2 d^{2} \alpha^{\prime} \cot \alpha\right), \\
h_{\mathcal{Y}}=-\frac{d}{1+d^{2}}\left(2 \alpha^{\prime} \cot \alpha+\frac{\beta_{1}^{\prime \prime}}{\beta_{1}^{\prime}}+\frac{\alpha^{\prime \prime}}{\alpha^{\prime}}\right) .
\end{gathered}
$$

Furthermore, (4.57) becomes

$$
(\gamma \tau)^{\prime}+2 \gamma \tau f_{\mathcal{X}}-(-1)^{r} \nu \gamma^{2} \tau=0 \Longrightarrow \gamma \tau=\left(\alpha^{\prime}\right)^{2} e^{(-1)^{r} \nu\left(1+d^{2}\right) \beta_{1}}
$$

So

$$
\tau=\frac{\left(\alpha^{\prime}\right)^{2} e^{(-1)^{r} \nu\left(1+d^{2}\right) \beta_{1}}}{\left(1+d^{2}\right) \beta_{1}^{\prime}}
$$

Note that (4.58) and (4.59) are implied by

$$
\left(f_{\mathcal{X}}-(-1)^{r} \gamma \nu\right) \varphi+\varphi^{\prime}-\alpha_{1} \gamma \tau=0 \Longrightarrow \alpha_{1}=\frac{\alpha^{\prime} \varphi^{\prime}-\left(\alpha^{\prime \prime}+(-1)^{r} \nu\left(1+d^{2}\right) \alpha^{\prime} \beta_{1}^{\prime}\right) \varphi}{\left(\alpha^{\prime}\right)^{3} e^{(-1)^{r} \nu\left(1+d^{2}\right) \beta_{1}}}
$$

Observe that

$$
\begin{aligned}
& \mathcal{U}=-\frac{\alpha^{\prime \prime}}{\alpha^{\prime}} \mathcal{X}-\alpha^{\prime}(\mathcal{Y}+d \mathcal{Z})+\frac{\alpha^{\prime} \varphi^{\prime}-\left(\alpha^{\prime \prime}+(-1)^{r} \nu\left(1+d^{2}\right) \alpha^{\prime} \beta_{1}^{\prime}\right) \varphi}{\left(\alpha^{\prime}\right)^{3} e^{(-1)^{r} \nu\left(1+d^{2}\right) \beta_{1}}} \\
& +\left(\varphi-\left(\alpha^{\prime}\right)^{2} e^{(-1)^{r} \nu\left(1+d^{2}\right) \beta_{1}} \mathcal{X}\right) \zeta_{r}, \\
& \mathcal{V}=\frac{1}{1+d^{2}}\left(2 d^{2} \alpha^{\prime}(\mathcal{Y}+d \mathcal{Z}) \cot \alpha+\left(\left(d^{2}-1\right) \mathcal{Y}-2 d \mathcal{Z}\right) \frac{\beta_{1}^{\prime \prime}}{2 \beta_{1}^{\prime}}+\left(d^{2} \mathcal{Y}-d \mathcal{Z}\right) \frac{\alpha^{\prime \prime}}{\alpha^{\prime}}\right) \\
& +\alpha^{\prime}(\mathcal{X}-d \mathcal{Z} \cot \alpha)+\frac{\left(\alpha^{\prime}\right)^{2} e^{(-1)^{r} \nu\left(1+d^{2}\right) \beta_{1}}}{\left(1+d^{2}\right) \sqrt{\beta_{1}^{\prime}}} \xi_{r}, \\
& \mathcal{W}=\frac{1}{1+d^{2}}\left(\left(\left(1-d^{2}\right) \mathcal{Z}-2 d \mathcal{Y}\right) \frac{\beta_{1}^{\prime \prime}}{2 \beta_{1}^{\prime}}+(\mathcal{Z}-d \mathcal{Y}) \frac{\alpha^{\prime \prime}}{\alpha^{\prime}}-2 d(\mathcal{Y}+d \mathcal{Z}) \alpha^{\prime} \cot \alpha\right) \\
& +d \alpha^{\prime}(\mathcal{X}+\mathcal{Y} \cot \alpha)+\frac{d\left(\alpha^{\prime}\right)^{2} e^{(-1)^{r} \nu\left(1+d^{2}\right) \beta_{1}}}{\left(1+d^{2}\right) \sqrt{\beta_{1}^{\prime}}} \xi_{r} .
\end{aligned}
$$


Moreover,

$$
\begin{gathered}
R_{1}=\left(4 b \delta_{0, r}+c \delta_{1, r}\right) c\left(\alpha^{\prime}\right)^{2} e^{(-1)^{r} \nu\left(1+d^{2}\right) \beta_{1}}\left[\left(\alpha^{\prime}\right)^{2} e^{(-1)^{r} \nu\left(1+d^{2}\right) \beta_{1}} \mathcal{X}-\varphi\right] \\
-\frac{\left(2\left(\alpha^{\prime \prime}\right)^{2}-\alpha^{\prime \prime}\right) \mathcal{X}}{\left(\alpha^{\prime}\right)^{2}}-\frac{2\left(\alpha^{\prime}\right)^{3} e^{(-1)^{r} \nu\left(1+d^{2}\right) \beta_{1}}}{\sqrt{\beta_{1}^{\prime}}} \xi_{r}-\left(\alpha^{\prime}\right)^{2}\left(1+d^{2}\right) \mathcal{X} \\
+d\left(\left(\alpha^{\prime}\right)^{2} \cot \alpha-\alpha^{\prime \prime}\right) \mathcal{Z}-\left(\alpha^{\prime \prime}+\left(d \alpha^{\prime}\right)^{2} \cot \alpha\right) \mathcal{Y}, \\
R_{2}=\gamma_{1}\left[\frac{\alpha^{\prime}\left(2 \alpha^{\prime \prime} \beta_{1}^{\prime}-\alpha^{\prime} \beta_{1}^{\prime \prime}\right) e^{(-1)^{r} \nu\left(1+d^{2}\right) \beta_{1}}}{\left(1+d^{2}\right)\left(\beta_{1}^{\prime}\right)^{2}} \xi_{r}+\frac{\left(\alpha^{\prime}\right)^{4} e^{(-1)^{r} 2 \nu\left(1+d^{2}\right) \beta_{1}}}{\left(1+d^{2}\right) \beta_{1}^{\prime}} \xi_{r} \zeta_{r}\right] \\
-\left(\alpha^{\prime \prime}+\left(d \alpha^{\prime}\right)^{2} \cot \alpha\right) \mathcal{X}+\left[k_{\mathcal{Y} t}-\left(\alpha^{\prime}\right)^{2}\left(1+d^{2} \csc ^{2} \alpha\right)\right] \mathcal{Y}+\frac{1}{2}\left(k^{2}+h^{2}\right) \mathcal{Y} \\
+\left[\left(k_{\mathcal{Z}}-\beta^{\prime} \cos \alpha\right)^{\prime}-d\left(\alpha^{\prime}\right)^{2}\right] \mathcal{Z}+2 \alpha^{\prime} f^{*}-2\left(\alpha^{\prime}\right)^{3} e^{(-1)^{r} \nu\left(1+d^{2}\right) \beta_{1}} \zeta_{r} \mathcal{X}, \\
R_{3}=\gamma_{2}\left[\frac{\alpha^{\prime}\left(2 \alpha^{\prime \prime} \beta_{1}^{\prime}-\alpha^{\prime} \beta_{1}^{\prime \prime}\right) e^{(-1)^{r} \nu\left(1+d^{2}\right) \beta_{1}}}{\left(1+d^{2}\right)\left(\beta_{1}^{\prime}\right)^{2}} \xi_{r}+\frac{\left(\alpha^{\prime}\right)^{4} e^{(-1)^{r} 2 \nu\left(1+d^{2}\right) \beta_{1}}}{\left(1+d^{2}\right) \beta_{1}^{\prime}} \xi_{r} \zeta_{r}\right] \\
+d\left(\left(\alpha^{\prime}\right)^{2} \csc \alpha-\alpha^{\prime \prime}\right) \mathcal{X}+\left(\left(h \mathcal{Y}+\beta^{\prime} \cos \alpha\right)^{\prime}-d\left(\alpha^{\prime}\right)^{2}\right) \mathcal{Y}+\frac{1}{2}\left(k^{2}+h^{2}\right) \mathcal{Z} \\
+\left(h_{\mathcal{Z} t}-\left(d \alpha^{\prime}\right)^{2} \csc ^{2} \alpha\right) \mathcal{Z}+2 d \alpha^{\prime} f^{*}-2 d\left(\alpha^{\prime}\right)^{3} e^{(-1)^{r} \nu\left(1+d^{2}\right) \beta_{1}} \zeta_{r} \mathcal{X} .
\end{gathered}
$$

By (3.12),

$$
\begin{aligned}
& p=\rho\left\{\frac{2\left(\alpha^{\prime}\right)^{3} e^{(-1)^{r} \nu\left(1+d^{2}\right) \beta_{1}} \xi_{r} \mathcal{X}}{\sqrt{\beta_{1}^{\prime}}}+\frac{\left(\alpha^{\prime}\right)^{2}}{2}\left(\mathcal{Y}^{2}+d^{2} \csc ^{2} \alpha\left(\mathcal{Y}^{2}+\mathcal{Z}^{2}\right)+2 d \mathcal{Y Z}\right)-\frac{2 \alpha^{\prime} \varphi \zeta_{r}}{\sqrt{\beta_{1}^{\prime}}}\right. \\
& +\frac{\left(2\left(\alpha^{\prime \prime}\right)^{2}-\alpha^{\prime \prime \prime}\right) \mathcal{X}^{2}}{2\left(\alpha^{\prime}\right)^{2}}+\frac{\alpha^{\prime} \alpha^{\prime \prime \prime}-\left(\alpha^{\prime \prime}\right)^{2}}{2\left(1+d^{2}\right) \alpha^{\prime}}\left(2 d \mathcal{Y Z}-d^{2} \mathcal{Y}^{2}-\mathcal{Z}^{2}\right)-\frac{\left(\alpha^{\prime}\right)^{4} e^{(-1)^{r} 2 \nu\left(1+d^{2}\right) \beta_{1}} \xi_{r}^{2}}{2\left(1+d^{2}\right) \beta_{1}^{\prime}} \\
& +\frac{(-1)^{r} \alpha^{\prime}\left(\alpha^{\prime} \beta_{1}^{\prime \prime}-2 \alpha^{\prime \prime} \beta_{1}^{\prime}\right) \zeta_{r}}{\left(1+d^{2}\right)\left(\beta_{1}^{\prime}\right)^{2} e^{(-1)^{r+1} \nu\left(1+d^{2}\right) \beta_{1}}}+\frac{2 \alpha^{\prime}\left[\left(\alpha^{\prime \prime}+(-1)^{r} \nu\left(1+d^{2}\right) \alpha^{\prime} \beta_{1}^{\prime}\right) \varphi-\alpha^{\prime} \varphi^{\prime}\right](\mathcal{Y}+d \mathcal{Z})}{\left(\alpha^{\prime}\right)^{3} e^{(-1)^{r} \nu\left(1+d^{2}\right) \beta_{1}}} \\
& -\frac{1}{2\left(1+d^{2}\right)^{2}}\left[\left(2 d^{2} \alpha^{\prime}(\mathcal{Y}+d \mathcal{Z} \cot \alpha)+\frac{\beta_{1}^{\prime \prime}\left(\left(d^{2}-1\right) \mathcal{Y}-2 d \mathcal{Z}\right)}{2 \beta_{1}^{\prime}}+\frac{\alpha^{\prime \prime}\left(d^{2} \mathcal{Y}-d \mathcal{Z}\right)}{\alpha^{\prime}}\right)^{2}\right. \\
& \left.+\left(\frac{\beta_{1}^{\prime \prime}\left(\left(1-d^{2}\right) \mathcal{Z}-2 d \mathcal{Y}\right)}{2 \beta_{1}^{\prime}}+\frac{\alpha^{\prime \prime}(\mathcal{Z}-d \mathcal{Y})}{\alpha^{\prime}}-2 d(\mathcal{Y}+d \mathcal{Z}) \alpha^{\prime} \cot \alpha\right)^{2}\right]-\frac{\left(\alpha^{\prime}\right)^{2}\left(1+d^{2}\right) \mathcal{X}^{2}}{2} \\
& +\frac{d}{1+d^{2}}\left(\alpha^{\prime \prime} \cot \alpha-\left(\alpha^{\prime}\right)^{2} \csc ^{2} \alpha\right)\left(\left(1-d^{2}\right) \mathcal{Y Z}+2 d\left(\mathcal{Z}^{2}-\mathcal{Y}^{2}\right)\right)+\frac{\beta_{1}^{\prime} \beta_{1}^{\prime \prime}-\left(\beta_{1}^{\prime \prime}\right)^{2}}{4\left(1+d^{2}\right)\left(\beta_{1}^{\prime}\right)^{2}} \\
& \times\left(4 d \mathcal{Y Z}+\left(1-d^{2}\right)\left(\mathcal{Y}^{2}-\mathcal{Z}^{2}\right)\right)+\left(\alpha^{\prime \prime}+\left(d \alpha^{\prime}\right)^{2} \cot \alpha\right) \mathcal{X} \mathcal{Y}+d\left(\alpha^{\prime \prime}-\left(\alpha^{\prime}\right)^{2} \cot \alpha\right) \mathcal{X} \mathcal{Z} \\
& \left.+\left(4 b \delta_{0, r}+c \delta_{1, r}\right) c\left(\alpha^{\prime}\right)^{2} e^{(-1)^{r} \nu\left(1+d^{2}\right) \beta_{1}}\left[\varphi \mathcal{X}-2^{-1}\left(\alpha^{\prime}\right)^{2} e^{(-1)^{r} \nu\left(1+d^{2}\right) \beta_{1}} \mathcal{X}^{2}\right]\right\}
\end{aligned}
$$

modulo the transformation in (1.10).

By (3.3) and (3.5), we have the following theorem:

Theorem 4.2. Let $\alpha, \beta_{1}, \varphi$ be functions of $t$ and let $b, c, d$ be real constants. Denote

$$
\beta=d \ln |\csc \alpha-\cot \alpha|
$$


(so the first equation in (4.63) holds). Define the moving frame $\mathcal{X}, \mathcal{Y}$ and $\mathcal{Z}$ by (3.1) and (3.5), and

$$
\begin{array}{ll}
\xi_{0}=b e^{\sqrt{\beta_{1}}(\mathcal{Y}+d \mathcal{Z})}-c e^{-\sqrt{\beta_{1}}(\mathcal{Y}+d \mathcal{Z})}, & \xi_{1}=c \sin \left[\sqrt{\beta_{1}}(\mathcal{Y}+d \mathcal{Z})+b\right], \\
\zeta_{0}=b e^{\sqrt{\beta_{1}}(\mathcal{Y}+d \mathcal{Z})}+c e^{-\sqrt{\beta_{1}}(\mathcal{Y}+d \mathcal{Z})}, & \zeta_{1}=c \cos \left[\sqrt{\beta_{1}}(\mathcal{Y}+d \mathcal{Z})+b\right] .
\end{array}
$$

For $r=0,1$, we have the following solution of the Navier-Stokes equations (1.1)-(1.4):

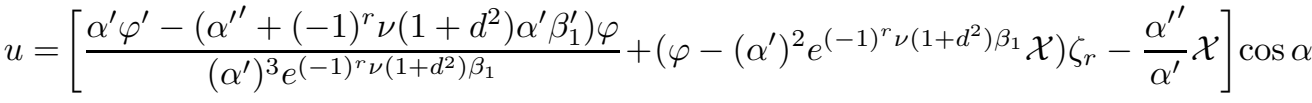

$$
\begin{aligned}
& -\frac{1}{1+d^{2}}\left(2 d^{2} \alpha^{\prime}(\mathcal{Y}+d \mathcal{Z}) \cot \alpha+\left(\left(d^{2}-1\right) \mathcal{Y}-2 d \mathcal{Z}\right) \frac{\beta_{1}^{\prime \prime}}{2 \beta_{1}^{\prime}}+\left(d^{2} \mathcal{Y}-d \mathcal{Z}\right) \frac{\alpha^{\prime \prime}}{\alpha^{\prime}}\right) \sin \alpha \\
& +\alpha^{\prime}(\mathcal{X} \sin \alpha-(\mathcal{Y}+2 d \mathcal{Z}) \cos \alpha)+\frac{\left(\alpha^{\prime}\right)^{2} e^{(-1)^{r} \nu\left(1+d^{2}\right) \beta_{1}}}{\left(1+d^{2}\right) \sqrt{\beta_{1}^{\prime}}} \xi_{r} \sin \alpha, \\
& v=\left[\frac{\alpha^{\prime} \varphi^{\prime}-\left(\alpha^{\prime \prime}+(-1)^{r} \nu\left(1+d^{2}\right) \alpha^{\prime} \beta_{1}^{\prime}\right) \varphi}{\left(\alpha^{\prime}\right)^{3} e^{(-1)^{r} \nu\left(1+d^{2}\right) \beta_{1}}}+\left(\varphi-\left(\alpha^{\prime}\right)^{2} e^{(-1)^{r} \nu\left(1+d^{2}\right) \beta_{1}} \mathcal{X}\right) \zeta_{r}\right] \sin \alpha \cos \beta \\
& +\frac{2 d \alpha^{\prime}(d \cos \alpha \cos \beta-\sin \beta)(\mathcal{Y}+d \mathcal{Z}) \cot \alpha}{1+d^{2}}+\frac{\alpha^{\prime \prime}(d \cos \alpha \cos \beta+\sin \beta)(d \mathcal{Y}-\mathcal{Z})}{\left(1+d^{2}\right) \alpha^{\prime}} \\
& +\frac{\left[\left(\left(d^{2}-1\right) \mathcal{Y}-2 d \mathcal{Z}\right) \cos \alpha \cos \beta-\left(\left(1-d^{2}\right) \mathcal{Z}-2 d \mathcal{Y}\right) \sin \beta\right] \beta_{1}^{\prime \prime}}{2\left(1+d^{2}\right) \beta_{1}^{\prime}}-\frac{\alpha^{\prime \prime}}{\alpha^{\prime}} \mathcal{X} \sin \alpha \cos \beta \\
& +\alpha^{\prime} \mathcal{X}(\cos \alpha \cos \beta-d \sin \beta)-\alpha^{\prime} \mathcal{Y}(\cos \alpha \cos \beta-d \cos \alpha \sin \beta) \\
& -d \alpha^{\prime} \mathcal{Z} \csc \alpha \cos \beta+\frac{\left(\alpha^{\prime}\right)^{2} e^{(-1)^{r} \nu\left(1+d^{2}\right) \beta_{1}}(\cos \alpha \cos \beta-d \sin \beta)}{\left(1+d^{2}\right) \sqrt{\beta_{1}^{\prime}}} \xi_{r}, \\
& w=\left[\frac{\alpha^{\prime} \varphi^{\prime}-\left(\alpha^{\prime \prime}+(-1)^{r} \nu\left(1+d^{2}\right) \alpha^{\prime} \beta_{1}^{\prime}\right) \varphi}{\left(\alpha^{\prime}\right)^{3} e^{(-1)^{r} \nu\left(1+d^{2}\right) \beta_{1}}}+\left(\varphi-\left(\alpha^{\prime}\right)^{2} e^{(-1)^{r} \nu\left(1+d^{2}\right) \beta_{1}} \mathcal{X}\right) \zeta_{r}\right] \sin \alpha \sin \beta \\
& +\frac{2 d \alpha^{\prime}(d \cos \alpha \sin \beta+\cos \beta)(\mathcal{Y}+d \mathcal{Z}) \cot \alpha}{1+d^{2}}+\frac{\alpha^{\prime \prime}(d \cos \alpha \sin \beta-\cos \beta)(d \mathcal{Y}-\mathcal{Z})}{\left(1+d^{2}\right) \alpha^{\prime}} \\
& +\frac{\left[\left(\left(d^{2}-1\right) \mathcal{Y}-2 d \mathcal{Z}\right) \cos \alpha \sin \beta+\left(\left(1-d^{2}\right) \mathcal{Z}-2 d \mathcal{Y}\right) \cos \beta\right] \beta_{1}^{\prime \prime}}{2\left(1+d^{2}\right) \beta_{1}^{\prime}}-\frac{\alpha^{\prime \prime}}{\alpha^{\prime}} \mathcal{X} \sin \alpha \sin \beta \\
& +\alpha^{\prime} \mathcal{X}(\cos \alpha \sin \beta+d \cos \beta)-\alpha^{\prime} \mathcal{Y}(\cos \alpha \sin \beta+d \cos \alpha \cos \beta) \\
& -d \alpha^{\prime} \mathcal{Z} \csc \alpha \sin \beta+\frac{\left(\alpha^{\prime}\right)^{2} e^{(-1)^{r} \nu\left(1+d^{2}\right) \beta_{1}}(\cos \alpha \sin \beta+d \cos \beta)}{\left(1+d^{2}\right) \sqrt{\beta_{1}^{\prime}}} \xi_{r},
\end{aligned}
$$

and $p$ is given in (4.81).

Case 2. $\gamma_{2}=\alpha_{1}=0,(\gamma \tau)^{\prime}+2 \gamma \tau f_{\mathcal{X}}-(-1)^{r} \nu \gamma^{2} \tau=0$ and $\gamma_{1} \neq 0$.

According to (4.51) and (4.54),

$$
k_{\mathcal{Y}}=-\frac{\gamma_{1}^{\prime}}{\gamma_{1}}, \quad k_{\mathcal{Z}}=0, \quad h_{\mathcal{Y}}=-2 \beta^{\prime} \cos \alpha .
$$


Note $\gamma=\gamma_{1}^{2}$. Moreover, (4.52) says

$$
k_{\mathcal{X}}=0, \quad h_{\mathcal{X}}=-2 \beta^{\prime} \sin \alpha .
$$

Furthermore, (4.61) yields

$$
f_{\mathcal{X}}=\frac{\gamma_{1}^{\prime}}{\gamma_{1}}
$$

Besides, (4.40) implies

$$
h_{\mathcal{Z}}=-\left(f_{\mathcal{X}}+k_{\mathcal{Y}}\right)=0 .
$$

Under the conditions (4.60) and (4.61), (4.58) and (4.59) are equivalent to

$$
f_{\mathcal{X}}=k_{\mathcal{Y}}=\gamma_{1}^{\prime}=0, \quad-(-1)^{r} \gamma \nu \varphi+\varphi^{\prime}=0 .
$$

Write $\gamma_{1}=a_{1}$ as a real constant. We have

$$
\sigma=a_{2} e^{(-1)^{r} \nu a_{1}^{2} t}, \quad \varphi=a_{1} e^{(-1)^{r} \nu a_{1}^{2} t}, \quad \tau=a_{1}^{-1} e^{(-1)^{r} \nu a_{1}^{2} t}
$$

for $a_{2} \in \mathbb{R}$ (cf. (4.47)). By (4.60)

$$
\beta^{\prime}= \pm \sqrt{\frac{-\alpha^{\prime \prime}}{\sin 2 \alpha}}
$$

that is,

$$
\beta= \pm \int \sqrt{\frac{-\alpha^{\prime \prime}}{\sin 2 \alpha}} d t
$$

Thus

$$
\begin{gathered}
\mathcal{U}=-\alpha^{\prime} \mathcal{Y}-\beta^{\prime} \mathcal{Z} \sin \alpha+a_{1} e^{(-1)^{r} \nu a_{1}^{2} t}\left(\vartheta_{r}+a_{2} \mathcal{Y} \phi_{r}-\zeta_{r} \mathcal{X}-a_{1} a_{2} \psi_{r} \mathcal{X}^{2}\right), \\
\mathcal{V}=\alpha^{\prime} \mathcal{X}-\beta^{\prime} \mathcal{Z} \cos \alpha+e^{(-1)^{r} \nu a_{1}^{2} t}\left(\xi_{r}+2 a_{1} a_{2} \phi_{r} \mathcal{X}\right), \quad \mathcal{W}=-\beta^{\prime}(\mathcal{X} \sin \alpha+\mathcal{Y} \cos \alpha) .
\end{gathered}
$$

Moreover,

$$
\begin{aligned}
R_{1} & =-\frac{\alpha^{\prime \prime}}{2} \mathcal{Y}+a_{1}^{2} e^{(-1)^{r} 2 \nu a_{1}^{2} t}\left[\left(4 b \delta_{0, r}+c \delta_{1, r}\right) c \mathcal{X}+3 a_{2}\left(2 \delta_{0, r}(a b+c)+\delta_{1, r} c \cos b\right) \mathcal{X}^{2}\right. \\
& +2 a_{1}^{2} a_{2}^{2}\left(4 a \delta_{0, r}+\delta_{1, r}\right) \mathcal{X}^{3}+a_{2}\left(2 \delta_{0, r}(a b-c)+\delta_{1, r} c \sin b\right) \mathcal{Y}+\delta_{1, r} c c_{1} \sin \left(b-b_{1}\right) \\
& \left.+2 \delta_{0, r}\left(b c_{1}-b_{1} c\right)+a_{1} a_{2}\left(2 \delta_{0, r}\left(c_{1}-a b_{1}\right)-\delta_{1, r} c_{1} \sin b_{1}\right) \mathcal{X}\right] \\
& +\left(\alpha^{\prime} \beta^{\prime} \cos \alpha-\left(\beta^{\prime} \sin \alpha\right)^{\prime}\right) \mathcal{Z}-\left(\left(\alpha^{\prime}\right)^{2}+\left(\beta^{\prime} \sin \alpha\right)^{2}\right) \mathcal{X} \\
& +e^{(-1)^{r} \nu a_{1}^{2} t}\left(a_{1} a_{2} \phi-2 \alpha^{\prime}\right)\left(\xi_{r}+2 a_{1} a_{2} e^{(-1)^{r} \nu a_{1}^{2} t} \phi_{r} \mathcal{X}\right) \\
R_{2}= & 2 a_{1} e^{(-1)^{r} \nu a_{1}^{2} t}\left[\left(\vartheta_{r}+a_{2} \mathcal{Y} \phi_{r}\right)\left(\alpha^{\prime}+a_{1} a_{2} e^{(-1)^{r} \nu a_{1}^{2} t} \phi_{r}\right)-\alpha^{\prime}\left(\zeta_{r} \mathcal{X}+a_{1} a_{2} \psi_{r} \mathcal{X}^{2}\right)\right] \\
- & \frac{\alpha^{\prime \prime}}{2} \mathcal{X}+\left(3\left(\beta^{\prime}\right)^{2} \cos ^{2} \alpha-\left(\alpha^{\prime}\right)^{2}\right) \mathcal{Y}-\left(\left(\beta^{\prime} \cos \alpha\right)^{\prime}+\alpha^{\prime} \beta^{\prime} \sin \alpha\right) \mathcal{Z} \\
+ & a_{1} e^{(-1)^{r} 2 \nu a_{1}^{2} t}\left(\xi_{r} \zeta_{r}+2 a_{1} a_{2} \xi_{r} \psi_{r} \mathcal{X}+2 a_{1}^{2} a_{2}^{2} \phi_{r} \psi_{r} \mathcal{X}^{2}\right) \\
R_{3} & =\left(\alpha^{\prime} \beta^{\prime} \cos \alpha-\left(\beta^{\prime} \sin \alpha\right)^{\prime}\right) \mathcal{X}-\left(\left(\beta^{\prime} \cos \alpha\right)^{\prime}+\alpha^{\prime} \beta^{\prime} \sin \alpha\right) \mathcal{Y}+\alpha^{\prime \prime} \mathcal{Z} \csc 2 \alpha
\end{aligned}
$$


By (3.12),

$$
\begin{aligned}
p & =\rho\left\{\frac{\alpha^{\prime \prime} \mathcal{X Y}}{2}-a_{1}^{2} e^{(-1)^{r} 2 \nu a_{1}^{2} t}\left[\frac{\left(4 b \delta_{0, r}+c \delta_{1, r}\right) c \mathcal{X}^{2}}{2}+a_{2}\left(2 \delta_{0, r}(a b+c)+\delta_{1, r} c \cos b\right) \mathcal{X}^{3}\right.\right. \\
& +a_{1}^{2} a_{2}^{2}\left(4 a \delta_{0, r}+\delta_{1, r}\right) \frac{\mathcal{X}^{4}}{2}+a_{2}\left(2 \delta_{0, r}(a b-c)+\delta_{1, r} c \sin b\right) \mathcal{X} \mathcal{Y}+\left(\delta_{1, r} c c_{1} \sin \left(b-b_{1}\right)\right. \\
& \left.\left.+2 \delta_{0, r}\left(b c_{1}-b_{1} c\right)\right) \mathcal{X}+\frac{a_{1} a_{2}\left(2 \delta_{0, r}\left(c_{1}-a b_{1}\right)-\delta_{1, r} c_{1} \sin b_{1}\right) \mathcal{X}^{2}}{2}\right]+\frac{\left(\left(\alpha^{\prime}\right)^{2}+\left(\beta^{\prime} \sin \alpha\right)^{2}\right) \mathcal{X}^{2}}{2} \\
& +\left(\left(\beta^{\prime} \sin \alpha\right)^{\prime}-\alpha^{\prime} \beta^{\prime} \cos \alpha\right) \mathcal{X} \mathcal{Z}-e^{(-1)^{r} \nu a_{1}^{2} t}\left(a_{1} a_{2} \phi-2 \alpha^{\prime}\right)\left(\xi_{r} \mathcal{X}+a_{1} a_{2} e^{(-1)^{r} \nu a_{1}^{2} t} \phi_{r} \mathcal{X}^{2}\right) \\
& -(-1)^{r} e^{(-1)^{r} \nu a_{1}^{2} t}\left[a _ { 2 } e ^ { ( - 1 ) ^ { r } \nu a _ { 1 } ^ { 2 } t } \left[a_{1} a_{2} \mathcal{Y} \phi_{r} \psi_{r}-2^{-1} a_{2}\left(a_{1}\left(4 a \delta_{0,1}+\delta_{1, r}\right) \mathcal{Y}^{2}+\phi_{r}^{2}\right)\right.\right. \\
& \left.\left.+\vartheta_{r} \zeta_{r}-\left(\delta_{0,1} 2\left(a b_{1}+c_{1}\right)+\delta_{1, r} c_{1}\right) \mathcal{Y}\right]+\alpha^{\prime}\left(\varepsilon_{r}+a_{2} \mathcal{Y} \psi_{r}-a_{1}^{-1} a_{2} \phi_{r}\right)\right]-\frac{e^{(-1)^{r} 2 \nu a_{1}^{2} t} \xi_{r}^{2}}{2} \\
& \left.-\frac{\left(3\left(\beta^{\prime}\right)^{2} \cos ^{2} \alpha-\left(\alpha^{\prime}\right)^{2}\right) \mathcal{Y}^{2}}{2}+\left(\left(\beta^{\prime} \cos \alpha\right)^{\prime}+\alpha^{\prime} \beta^{\prime} \sin \alpha\right) \mathcal{Y Z}-\frac{\alpha^{\prime \prime} \mathcal{Z}^{2} \csc 2 \alpha}{2}\right\}
\end{aligned}
$$

modulo the transformation in (1.10), where

$$
\varepsilon_{0}=b_{1} e^{\tilde{\omega}}+c_{1} e^{-\tilde{\omega}}, \quad \varepsilon_{1}=c_{1} \cos \left(\tilde{\varpi}+b_{1}\right)
$$

in connection with $(4.56)$ and $\tilde{\varpi}=a_{1} \mathcal{Y}$.

By (3.3) and (3.5), we have the following theorem:

TheOREM 4.3. Let $\alpha$ be a function of $t$ and let $a, a_{1}, a_{2}, b, b_{1}, c, c_{1}$ be real constants. Denote $\beta$ as in (4.91). Define the moving frame $\mathcal{X}, \mathcal{Y}$ and $\mathcal{Z}$ by (3.1) and (3.5), and

$$
\begin{gathered}
\phi_{0}=e^{a_{1} \mathcal{Y}}-a e^{-a_{1} \mathcal{Y}}, \quad \phi_{1}=\sin \left(a_{1} \mathcal{Y}\right), \quad \psi_{0}=e^{a_{1} \mathcal{Y}}+a e^{-a_{1} \mathcal{Y}}, \quad \psi_{1}=\cos \left(a_{1} \mathcal{Y}\right), \\
\xi_{0}=b e^{a_{1} \mathcal{Y}}-c e^{-a_{1} \mathcal{Y}}, \quad \xi_{1}=c \sin \left(a_{1} \mathcal{Y}+b\right), \quad \zeta_{0}=b e^{a_{1} \mathcal{Y}}+c e^{-a_{1} \mathcal{Y}}, \\
\zeta_{1}=c \cos \left(a_{1} \mathcal{Y}+b\right), \quad \vartheta_{0}=b_{1} e^{a_{1} \mathcal{Y}}-c_{1} e^{-a_{1} \mathcal{Y}}, \quad \vartheta_{1}=c_{1} \sin \left(a_{1} \mathcal{Y}+b_{1}\right), \\
\varepsilon_{0}=b_{1} e^{a_{1} \mathcal{Y}}+c_{1} e^{-a_{1} \mathcal{Y}}, \quad \varepsilon_{1}=c_{1} \cos \left(a_{1} \mathcal{Y}+b_{1}\right) .
\end{gathered}
$$

For $r=0,1$, we have the following solution of the Navier-Stokes equations (1.1)-(1.4):

$$
\begin{gathered}
u=\left[-\alpha^{\prime} \mathcal{Y}+a_{1} e^{(-1)^{r} \nu a_{1}^{2} t}\left(\vartheta_{r}+a_{2} \mathcal{Y} \phi_{r}-\zeta_{r} \mathcal{X}-a_{1} a_{2} \psi_{r} \mathcal{X}^{2}\right)\right] \cos \alpha \\
-\left[\alpha^{\prime} \mathcal{X}+e^{(-1)^{r} \nu a_{1}^{2} t}\left(\xi_{r}+2 a_{1} a_{2} \phi_{r} \mathcal{X}\right)\right] \sin \alpha \\
v=\left[-\alpha^{\prime} \mathcal{Y}+a_{1} e^{(-1)^{r} \nu a_{1}^{2} t}\left(\vartheta_{r}+a_{2} \mathcal{Y} \phi_{r}-\zeta_{r} \mathcal{X}-a_{1} a_{2} \psi_{r} \mathcal{X}^{2}\right)\right] \sin \alpha \cos \beta-\beta^{\prime} \mathcal{Z} \cos \beta \\
-\left[\alpha^{\prime} \mathcal{X}+e^{(-1)^{r} \nu a_{1}^{2} t}\left(\xi_{r}+2 a_{1} a_{2} \phi_{r} \mathcal{X}\right)\right] \cos \alpha \cos \beta+\beta^{\prime}(\mathcal{X} \sin \alpha+\mathcal{Y} \cos \alpha) \sin \beta \\
w=\left[-\alpha^{\prime} \mathcal{Y}+a_{1} e^{(-1)^{r} \nu a_{1}^{2} t}\left(\vartheta_{r}+a_{2} \mathcal{Y} \phi_{r}-\zeta_{r} \mathcal{X}-a_{1} a_{2} \psi_{r} \mathcal{X}^{2}\right)\right] \sin \alpha \sin \beta-\beta^{\prime} \mathcal{Z} \sin \beta \\
-\left[\alpha^{\prime} \mathcal{X}+e^{(-1)^{r} \nu a_{1}^{2} t}\left(\xi_{r}+2 a_{1} a_{2} \phi_{r} \mathcal{X}\right)\right] \cos \alpha \sin \beta-\beta^{\prime}(\mathcal{X} \sin \alpha+\mathcal{Y} \cos \alpha) \cos \beta,
\end{gathered}
$$

and $p$ is given in (4.101). 


\section{REFERENCES}

[BK] M. A. Brutyan and P. L. Krapivsky, Exact solutions of Navier-Stokes equations describing the evolution of a vortex structure in generalized shear flow, Comput. Math. Phys. 32 (1992), 270272. MR 1166987 (93f:76022)

[Ba] A. A. Buchnev, Lie group admitted by the equations of motion of an ideal incompressible fluid, Dinamika Sploshnoi Sredi. Int. of Hydrodynamics Novosibirsk 7 (1971), 212.

[Bv1] V. O. Bytev, Nonsteady motion of a rotating ring of viscous incompressible fluid with free boundary, Zhumal Prikladnoi Mekhaniki i Tekhnicheskoi Fiziki 3 (1970), 83. MR0285187 (44:2410)

[Bv2] V. O. Bytev, Invariant solutions of the Navier-Stokes equations, Zhumal Prikladnoi Mekhaniki $i$ Tekhnicheskoi Fiziki 6 (1972), 56.

[G] V. I. Gryn, Exact solutions of Navier-Stokes equations, J. Appl. Math. Mech. 55 (1991), 301-309. MR:1134603 (92m:76042)

[I] N. H. Ibragimov, Lie Group Analysis of Differential Equations, Volume 2, CRC Handbook, CRC Press, 1995.

[J] G. B. Jeffery, Philosophical Magazine, Ser. 6 (1915), 29.

$[\mathrm{K}] \quad$ L. B. Kapitanskii, Group analysis of Navier-Stokes equations and Euler equations with rotational symmetry and new exact solutions of these equations, Dokl. Akad. Nauk S.S.S.R. 243 (1978), 901.

[KKR] H. E. Kochin, I. A. Kibel' and N. V. Roze, Theoretical Hydromechanics, Fizmatgiz, Moscow, 1963.

[Ll] L. Landau, A new exact solution of Navier-Stokes equations, C. R. (Doklady) Acad. Sci. URSS (N. S.) 43 (1944), 286-288. MR0011205 (6:135d)

[Lr] R. B. Leipnik, Exact solutions of Navier-Stokes equations by recursive series of diffusive equations, C. R. Math. Rep. Acad. Sci. Canada 18 (1996), 211-216. MR1425294 (97i:76039)

[LRT] C. C. Lin, E. Reissner and H. S. Tsien, On two-dimensional non-steady motion of a slender body in a compressible fluid, J. Math. Phys. 27 (1948), no. 3, 220. MR0026499 (10:162e)

[O] L. V. Ovsiannikov, Group Analysis of Differential Equations, Academic Press, New York, 1982. MR668703 (83m:58082)

[Pa] A. D. Polyanin, Exact solutions of the Navier-Stokes equations with generalized separation of variables, Dokl. Phys. 46 (2001), 726-731. MR.1875505 (2002g:76039)

[Pv1] V. V. Pukhnachev, Group properties of Navier-Stokes equations in two-dimensional case, Zhumal Prikladnoi Mekhaniki i Tekhnicheskoi Fiziki 1 (1960), 83.

[Pv2] V. V. Pukhnachev, Invariant solutions of Navier-Stokes equations describing motions with free boundary, Dokl. Akad. Nauk S.S.S.R. 202 (1972), 302.

[S1] H.-C. Shen, The theory of functions of a complex variable under Dirac-Pauli representation and its application in fluid dynamics I, Appl. Math. Mech. (English Ed.) 7 (1986), 391-411. MR861144 (88d:30057)

[S2] H.-C. Shen, Exact solutions of Navier-Stokes equations - the theory of functions of a complex variable under Dirac-Pauli representation and its application in fluid dynamics II, Appl. Math. Mech. (English Ed.) 7 (1986), 557-562. MR866002(88j:35129)

[V] V. G. Vyskrebtsov, New exact solutions of Navier-Stokes equations for axisymmetric self-similar fluid flows, J. Math. Sci. (New York) 104 (2001), 1456-1463. MR.1706756 (2000d:76042)

[W] Z. Warsi, Fluid Dynamics, CRC Press LTC, 1999. MR.1658211 (99k:76001)

[X1] X. Xu, Stable-range approach to the equation of nonstationary transonic gas flows, Quart. Appl. Math 65 (2008), 529-547. MR2354886

[X2] X. Xu, Parameter-function approach to classical non-steady boundary Layer problems, arXiv:0706.1864.

[Y] A. Yu. Yakimov, Exact solutions of Navier-Stokes equations in the presence of a vortex singularity on a ray, Dokl. Acad. Nauk SSSR 276 (1984), 79-82. MR.744894 (85e:76020) 